TRANSACTIONS OF THE

AMERICAN MATHEMATICAL SOCIETY

Volume 359, Number 5, May 2007, Pages 2075-2108

S 0002-9947(06)03949-3

Article electronically published on December 20, 2006

\title{
GEOMETRIC INTERPLAY BETWEEN FUNCTION SUBSPACES AND THEIR RINGS OF DIFFERENTIAL OPERATORS
}

\author{
RIKARD BÖGVAD AND ROLF KÄLLSTRÖM
}

\begin{abstract}
We study, in the setting of algebraic varieties, finite-dimensional spaces of functions $V$ that are invariant under a ring $\mathcal{D}^{V}$ of differential operators, and give conditions under which $\mathcal{D}^{V}$ acts irreducibly. We show how this problem, originally formulated in physics, is related to the study of principal parts bundles and Weierstrass points, including a detailed study of Taylor expansions. Under some conditions it is possible to obtain $V$ and $\mathcal{D}^{V}$ as global sections of a line bundle and its ring of differential operators. We show that several of the published examples of $\mathcal{D}^{V}$ are of this type, and that there are many more - in particular, arising from toric varieties.
\end{abstract}

\section{INTRODUCTION}

Assume that $V$ is a finite-dimensional vector subspace of a ring of functions $A$. The problem to describe properties of the ring of differential operators $\mathcal{D}_{A}^{V}$ on $A$ that preserve $V$, which is studied in this paper, has its origin in quantum mechanics. Such invariant subspaces form the foundation of the theory of quasi-exactly solvable quantum models, as formulated by Turbiner, Shifman, Ushveridze et al. (see the survey [11] and the introduction to [21], as well as the references given there).

It is easy to see for the polynomial algebra $A=\mathbf{C}\left[x_{1}, \ldots, x_{n}\right]$, that $\mathcal{D}_{A}^{V}$ always acts irreducibly on $V$ (see 29] for the one-variable case; the general case follows from the density theorem since $A$ is an irreducible $\mathcal{D}_{A}$-module), and a similar result was proved using different methods for real-analytic functions on a domain of some $\mathbf{R}^{n}$ by Kamran, Milson and Olver in 21. The latter authors introduce a condition on $V$-regularity - that ensures that $\mathcal{D}_{A}^{V}$ acts irreducibly on $V$.

There is also a Lie-algebraic approach, by directly finding realizations of a Lie algebra as differential operators on a (polynomial) ring preserving a vector space [21, 30. It was noted in [10] that some of these examples stem from a global variety with a line bundle. An acquaintance with [1, 3] makes it evident that many examples actually are local descriptions of rings of global differential operators of line bundles on homogeneous spaces or toric varieties.

Thus there is an interesting global slant to the problem of finding invariant subspaces. To understand it and also to extend the results of [21] to spaces other than open subsets of $\mathbf{R}^{n}$, it is natural to study invariant subspaces in the setting of sheaves of functions. Then the same global object will unite many different

Received by the editors June 8, 2004 and, in revised form, February 5, 2005.

2000 Mathematics Subject Classification. Primary 14F05, 58J99; Secondary 14L30.

(C)2006 American Mathematical Society Reverts to public domain 28 years from publication 
embeddings of $V$ in different rings of functions. The working out of the details of this more powerful and flexible way of approaching invariant subspaces, and exemplifying it, is the main contribution of the present paper.

In [14 differential operators are defined using bundles of principal parts. This point of view greatly simplifies the analysis of regularity. Our approach has the added advantage that it makes the results on points of Weierstrass subvarieties (points of inflexion and osculating planes) by Laksov and Thorup 23. available for the study of differential operators. Rather to our surprise it turns out that some of the ideas and results are the same; for example, the concept of regularity from 21. is also treated in [23. It will, moreover, be clear that on a nonsingular variety in characteristic 0 the two natural setups, one being based on the sheaf of principal parts, the other on differential operators, are in perfect correspondence.

We will now describe the content of the paper in more detail. Central to our whole approach is the Taylor map

$$
\mathrm{d}_{V}^{n}: \mathcal{O}_{X} \otimes_{k} V \rightarrow \mathcal{P}_{X / k}^{n}(M)
$$

to the bundle of principal parts of a locally free $\mathcal{O}_{X}$-module $M$ (this will give matrix-valued differential operators), where $V$ is a subspace of $\Gamma(X, M)$; this is studied in Chapter 2. We are first interested whether there exists an integer $n_{i n j}(x)$ giving fibrewise injectivity $\mathrm{d}_{V, x}^{n}: k_{x} \otimes_{k} V \rightarrow k_{x} \otimes_{\mathcal{O}_{x}} \mathcal{P}_{X / k}^{n}(M)$ when $n \geq n_{i n j}(x)$, the separability of $V$. Using Krull's theorem on the intersection of powers of an ideal we get simple criteria for separability in Theorem 2.2.5 and Corollary 2.2.7. Theorem 2.2.11 contains conditions guaranteeing the existence of $n_{i n j}$, such that $n \geq n_{\text {inj }}$ implies injectivity of the Taylor map at all points. These results extend by different means the conditions given in 23, inter alia from smooth to geometrically integral varieties, as well as bypass the complicated proof referred to in [21. Our analysis of separability generalises the latter authors' use of regularity; it reaches a more fundamental level, since we work with more general varieties. The surjectivity of the Taylor map is also useful: Define $n_{\text {surj }}\left(n_{\text {surj }}^{1}\right)$ if the Taylor map is injective at all points (points of height 1 ) for $n \leq n_{\text {surj }}$ (respectively $n_{\text {surj }}^{1}$ ); it is related to jet-ampleness of a line bundle.

Using the definition of differential operators of Grothendieck $\mathcal{D}_{X / k}(M)=$ $\longrightarrow \lim \left(\mathcal{P}_{X / k}^{n}(M) ; M\right)$, in Chapter 3 we apply the earlier results on the Taylor map to describe the action of differential operators on $V \subset \Gamma(X, M)$. In Theorem 3.2.1 the surjectivity of the evaluation map

$$
W_{x}^{n}: \mathcal{D}^{n}(M)_{x} \rightarrow \operatorname{Hom}_{k}\left(V, M_{x}\right)
$$

for differential operators on $M$ of order $n$ is shown to be equivalent to the injectivity of the Taylor map $\mathrm{d}_{V, x}^{n}$. In particular, if $V$ is separated, $W_{x}^{n}$ is surjective for high $n$ (Theorem 3.2.4). As a consequence the ring of differential operators $\mathcal{D}_{X / k}^{V}(M)$ that preserve $V \subset \Gamma(X, M)$ acts irreducibly if $X$ is affine (Theorem 3.3.3). This extends the algebraic counterpart of the main result of [21, Th. 4.8], bypassing Hodge algebra, from Zariski open subsets of $\mathbf{R}^{n}$ to real quasi-projective varieties, noting they are affine. The chapter begins by proving that if the Taylor map $\mathrm{d}_{V}^{n}$ is injective for some $n$, then it is actually injective for $n \geq|V|-1$ (Proposition 3.1.1), a result also contained in 23. Weierstrass subschemes $W(V)$ are defined using the semi-continuous function $x \mapsto n_{i n j}(x)$ (when $X / k$ is nonsingular); $W(V)$ was 
constructed in a different way in 23]. Our construction also gives a decreasing filtration of $W(V)$, and a candidate for a definition of Weierstrass subschemes on any scheme locally of finite type.

Up to this point our theory has been local. As mentioned above many of the interesting examples, in the sense that they give rise to physical models, arise from Lie algebras that somehow act as differential operators both on an affine variety and a finite-dimensional vector space. In [21, p. 316] the authors ask for an explanation of the "significant mystery [that] is the connection [of the algebraic approach of [21], with the Lie algebraic approach of [30] to quasi-exactly solvable modules". By inspection many of these examples are seen to be restrictions to an affine open subset $U$ from a global variety $X$ equipped with a line-bundle $\mathcal{L}$ (modulo the annihilator Ann $V$ ). In Chapters 5 and 6 we review homogeneous spaces and toric varieties, and by combining results in the literature with our setup it is immediate to see that both these classes of varieties produce subspaces $V \subset A:=\mathbf{C}\left[x_{1}, \ldots, x_{n}\right]$ such that $\mathcal{D}^{V}(\bmod \operatorname{Ann} V)$ arise as restrictions of the ring of global differential operators on an ample line bundle. Hence the Lie algebras arise from an invisible global context, while the approach of [21 is local. This explains, we believe the connection. It also makes it interesting to consider the question of when a vector space $V \subset \Gamma\left(X, \mathcal{O}_{X}\right)$ and its ring of differential operators $\mathcal{D}_{X}^{V}$ comes by restriction from a global context. There is an algebraic geometric technique, known to the ancients, to use $V$ to define a projective variety $X \subset X_{V}$ and a line-bundle $\mathcal{L}$ on $X_{V}$; in Chapter 4 we describe it and give in Theorem 4.2.1 conditions when we may extend differential operators $\mathcal{D}_{X}^{V}$ to differential operators on $X_{V}$ that preserve $\mathcal{L}$. This is used in Proposition 5.2.5 to prove that this machinery will detect "hidden symmetries": If $V \subset A$ is a finite-dimensional vector space such that there is a reductive Lie subalgebra $\mathfrak{g}$ of $\mathcal{D}_{A}$ that acts irreducibly on $V$ and locally transitively on $A$ with a parabolic subalgebra stabilising a point, then $\mathfrak{g}$ is the restriction of differential operators on $X_{V}$ that preserve $\mathcal{L}$.

Musson [25] describes the ring of global differential operators on a line bundle on a toric variety. In particular, it is not difficult to use this description to give many examples of smooth varieties where the ring of differential operators preserving a vector space $V$ is not generated by first order differential operators. These general results and toric constructions $X_{V}$, corresponding to a $V$ with a basis of monomials, do not seem to have been used before for the study of $\mathcal{D}^{V}$, even though Hirzebruch surfaces have been studied [12, 7].

We have further used both toric varieties and homogeneous spaces to exemplify our concepts, in particular we use the equivariant structure on the bundle of principal parts (Proposition 5.2.2) to determine (for toric varieties) and estimate (for homogeneous varieties) $n_{i n j}$, and in the case of Hirzebruch surfaces, we also make more explicit point-wise calculations.

We would like to thank T. Ekedahl and the referee for valuable comments.

Notations and assumptions: A variety $X / k$ is an irreducible scheme of finite type over a field, and $\mathcal{O}_{X}$ is its structure sheaf. If $x$ is a point on a scheme $X$ and $M$ a sheaf of modules over $\mathcal{O}_{X}$, we let $k_{x}$ be the residue field of $\mathcal{O}_{X}, M_{x}$ the stalk of $M$, and $k_{x} \otimes_{\mathcal{O}_{x}} M_{x}$ the fibre of $M$, at $x$. If $\phi: \mathcal{F} \rightarrow \mathcal{G}$ is a map of $\mathcal{O}_{X}$-modules, then $\phi_{x}: \mathcal{F}_{x} \rightarrow \mathcal{G}_{x}$ is the map of stalks and $\phi(x): k_{x} \otimes_{\mathcal{O}_{x}} \mathcal{F}_{x} \rightarrow k_{x} \otimes_{\mathcal{O}_{x}} \mathcal{G}_{x}$ the map of fibres at $x$. Throughout the paper the base field $k$ is of characteristic 0 and $M$ will denote a locally free $\mathcal{O}_{X}$-module of finite rank. 


\section{TAYLOR MAPS}

2.1. Generalities. A general reference for the material in this section is [14. Let $\left(X / k, \mathcal{O}_{X}\right)$ be a variety and $M$ a locally free $\mathcal{O}_{X}$-module of finite rank. Let $\Delta$ : $X \rightarrow X \times_{k} X, x \mapsto(x, x)$ be the diagonal map, $I_{\Delta}$ the kernel of the mapping $\Delta^{*}\left(\mathcal{O}_{X \times{ }_{k} X}\right) \rightarrow \mathcal{O}_{X}$, and put, for each integer $n \geq 0, \mathcal{P}_{X / k}^{n}=\Delta^{*}\left(\mathcal{O}_{X \times{ }_{k} X}\right) / I_{\Delta}^{n+1}$; define also $\mathcal{P}_{X / k}^{-1}=0$. The sheaf of $\left(\mathcal{O}_{X}, \mathcal{O}_{X}\right)$-bimodules $\mathcal{P}_{X / k}^{n}$ is the sheaf of $n$th order principal parts. Put also $\mathcal{P}_{X / k}^{n}(M)=\mathcal{P}_{X / k}^{n} \otimes_{\mathcal{O}_{X}} M$.

Let $V$ be a finite-dimensional $k$-subspace of the space of global sections $M(X):=$ $\Gamma(X, M) ; V$ is also regarded as a constant sheaf on $X$. There is a map $M \rightarrow$ $\mathcal{P}_{X / k}^{n}(M), m \mapsto 1 \otimes m$, which is injective since $M$ is locally free, and composing it with the injective map $V \rightarrow M$ we get an injective map $V \rightarrow \mathcal{P}_{X / k}^{n}(M)$. Putting $\mathcal{V}_{X}=\mathcal{O}_{X} \otimes_{k} V$ we get a map $\mathrm{d}_{V}^{n}: \mathcal{V}_{X} \rightarrow \mathcal{P}_{X / k}^{n}(M), \phi \otimes v \mapsto \phi \otimes v \bmod I_{\Delta}^{n+1}$; this map however need not be injective. Let $\mathcal{K}^{n}$ and $\mathcal{C}^{n}$ be the kernel and cokernel, respectively, of $\mathrm{d}_{V}^{n}$, so we have the exact sequence, which we will refer to as the Taylor sequence,

$$
0 \rightarrow \mathcal{K}^{n} \rightarrow \mathcal{V}_{X} \stackrel{\mathrm{d}_{V}^{n}}{\longrightarrow} \mathcal{P}_{X / k}^{n}(M) \rightarrow \mathcal{C}^{n} \rightarrow 0
$$

Varying the integer $n$ one gets different exact sequences (2.1), connected in the diagram

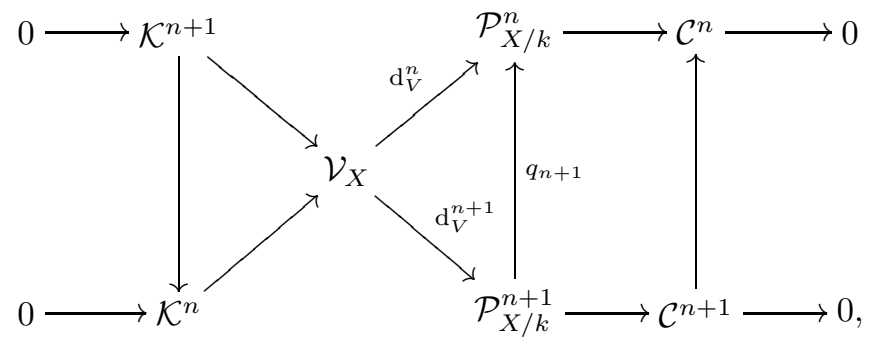

where $q_{n+1}$ is the natural projection map. The inverse limit

$$
\mathcal{P}_{X / k}^{\infty}(M):={\underset{\lim }{n}}_{\mathcal{P}_{X / k}^{n}}^{n}(M)
$$

is provided with the $I_{\Delta}$-adic topology, which is used to define the sheaf of differential operators as the $\mathcal{O}_{X}$-bimodule of continuous maps

$$
\mathcal{D}_{X}(M):=\operatorname{Hom}_{\mathcal{O}_{X}}^{\text {cont }}\left(\mathcal{P}_{X / k}^{\infty}(M), M\right)
$$

where $M$ is given the discrete topology. The $\mathcal{O}_{X}$-bimodule of differential operators of order at most $n$ is denoted $\mathcal{D}_{X}^{n}(M)$, which together for all $n$ give a filtration

$$
\begin{gathered}
\mathcal{D}_{X}^{n}(M):=\operatorname{Hom}_{\mathcal{O}_{X}}\left(\mathcal{P}_{X / k}^{n}(M), M\right), \\
0 \subset \mathcal{D}_{X}^{0}(M)=\operatorname{End}_{\mathcal{O}_{X}}(M) \subset \cdots \subset \mathcal{D}_{X}^{n}(M) \subset \mathcal{D}_{X}^{n+1}(M) \subset \cdots, \\
\mathcal{D}_{X}(M)=\bigcup_{n=0}^{\infty} \mathcal{D}_{X}^{n}(M) .
\end{gathered}
$$

The fibre of the sheaf of principal parts at a $k$-rational point $x$ is

$$
k \otimes_{\mathcal{O}_{x}} \mathcal{P}_{X / k}^{n}(M)_{x} \cong M_{x} / \mathfrak{m}_{x}^{n+1} M_{x} \quad([14,16.4 .11])
$$


and if the stalk $\mathcal{P}_{X / k}^{n}(M)_{x}$ is free, then the fibre $k \otimes_{\mathcal{O}_{x}} \mathcal{D}_{x}^{n}(M)=\left(M_{x} / \mathfrak{m}_{x}^{n+1} M_{x}\right)^{*}:=$ $\operatorname{Hom}_{k}\left(M_{x} / \mathfrak{m}_{x}^{n+1} M_{x}, k\right)$.

We will need that $\mathcal{P}_{X / k}^{n}(M)$ be locally free over $\mathcal{O}_{X}$ (of finite rank).

Lemma 2.1.1. A scheme of finite type $X / k$ is regular if and only if the $\mathcal{O}_{X}$-module $\mathcal{P}_{X / k}^{n}$ is locally free for each integer $n$.

Proof. If $X$ is regular, $X$ is smooth (note Char $k=0$ ), and $\Omega_{X / k}$ is locally free ([14, Cor. 17.5.2, 17.15.6]). Hence $\mathcal{P}_{X / k}^{n}$ is locally free $([14$, Thm. 16.12.2, Def.16.10.1]). The converse follows from the same reference.

Let

$$
\mathrm{d}_{V, x}^{n}: \mathcal{V}_{x} \rightarrow \mathcal{P}_{X / k, x}^{n}(M)
$$

be the map of stalks at $x$. This is the $n$th Taylor expansion map at $x$ of the vectors in $V$. In a smooth rational point $x$, there is a basis of $\mathcal{P}_{X / k, x}^{n}\left(\mathcal{O}_{X}\right)$, consisting of all monomials of degree less than $n$ in $\xi_{i}=x_{i} \otimes 1-1 \otimes x_{i}$, where $x_{i}$ are a regular system of parameters at $x$. Let the derivations $\partial_{1}, \ldots, \partial_{d}$ correspond to the coordinates. Then the map $\mathrm{d}_{V, x}^{n}$ is described by the matrix with rows $\left(\partial^{\alpha}\left(m_{i}\right) / \alpha !\right)$, for $m_{i}$ a basis of $V$. Here the multi-indices $\alpha=\left(\alpha_{1}, \ldots, \alpha_{d}\right)$ take all possible values that define a differential operator of order less than or equal to $n, \partial^{\alpha}=\prod\left(\partial / \partial x_{i}\right)^{\alpha_{i}}$ and $\alpha !=\prod \alpha_{i} !$

The map of fibres at a point $x$ is with residue field $k_{x}$ is

$$
\mathrm{d}_{V}^{n}(x): k_{x} \otimes_{k} V \rightarrow k_{x} \otimes_{\mathcal{O}_{x}} \mathcal{P}_{X / k, x}^{n}(M) .
$$

2.2. The injectivity of the Taylor map. The following elementary fact will play a central part in the paper [4, Proposition II.3.6].

Proposition 2.2.1. Let $\phi: \mathcal{F} \rightarrow \mathcal{G}$ be a map of locally free $\mathcal{O}_{X}$-modules of finite rank. Then the following are equivalent at a point $x$ in $X$ :

(1) the map of fibres $\phi(x): k_{x} \otimes_{\mathcal{O}_{x}} \mathcal{F}_{x} \rightarrow k_{x} \otimes_{\mathcal{O}_{x}} \mathcal{G}_{x}$ is injective;

(2) the map of stalks $\phi_{x}: \mathcal{F}_{x} \rightarrow \mathcal{G}_{x}$ is split injective.

We will employ Krull's theorem in order to get separated topologies on a Noetherian tensor product $K_{1} \otimes_{k} A$. Therefore we need $K_{1} \otimes_{k} A$ to be integral for all field extensions $K_{1}$ of $k$, so the following lemma is useful.

Lemma 2.2.2 ([14, Cor. 4.6.3]). Let $X / k$ be an integral scheme. The following are equivalent:

(1) $k$ is algebraically closed in the function field of $X$;

(2) $X \times_{k} K$ is integral for each extension $K$ of $k$.

One says that the scheme $X / k$ is geometrically integral if it satisfies these conditions. In particular, $\mathcal{O}_{x} \otimes_{k} K$ is always integral.

That the Taylor map is injective will turn out to have great significance for the properties of differential operators that we are interested in. We have an exact sequence

$$
0 \rightarrow \mathcal{K} \rightarrow \Delta^{*}\left(p_{2}^{*}(M)\right) \stackrel{\mathrm{d}}{\rightarrow} \mathcal{P}_{X / k}^{\infty}(M)
$$

where $\mathcal{K}=\bigcap_{n \geq 1} I_{\Delta}^{n} M$ and $p_{2}: X \times_{k} X \rightarrow X$ is the projection on the second factor; $I_{\Delta}^{n} M$ denotes the image of the canonical map $I_{\Delta}^{n+1} \otimes_{\mathcal{O}_{X}} M \rightarrow \Delta^{*}\left(p_{2}^{*}(M)\right.$ ) (which is injective when $\mathcal{P}_{X / k}^{n}$ is flat over $\left.\mathcal{O}_{X}\right)$. Hence $\mathrm{d}$ is injective if the $I_{\Delta}$-adic topology on the $\mathcal{O}_{X}$-module $\Delta^{*}\left(\mathcal{O}_{X \times_{k} X} \otimes_{\mathcal{O}_{X}} M\right)$ is separated. 
Proposition 2.2.3. Let $X / k$ be a scheme of finite type. If $X \times_{k} X$ is integral (e.g. $X / k$ is geometrically integral), then the $I_{\Delta}$-adic topology on $\Delta^{*}\left(p_{2}^{*}(M)\right)$ is separated, or equivalently, the Taylor map d is injective.

Proof. The map $\mathrm{d}$ is injective if it is injective at associated points of $X$. It therefore suffices to prove the following. Let $K / k$ be a field extension and $M$ be a finitedimensional linear space over $K$ and $I_{\Delta}$ be the kernel of $K \otimes_{k} K \rightarrow K, a \otimes b \mapsto a b$. Then the map $d_{K / k, M}: K \otimes_{k} M \rightarrow \mathcal{P}_{K / k}^{\infty}(M)=\lim _{n \rightarrow \infty} K \otimes K / I_{\Delta}^{n+1} \otimes_{K} M$ is injective. But $\operatorname{Ker}\left(K \otimes_{k} K \rightarrow \mathcal{P}_{K / k}^{\infty}\right)=\bigcap_{n \geq 1} I_{K}^{n}$ and $\operatorname{Ker}\left(K \otimes_{k} M \rightarrow \mathcal{P}_{K / k}^{\infty}(M)\right)=$ $\bigcap\left(I_{\Delta}^{n} M\right)=\left(\bigcap_{n \geq 1} I_{K}^{n}\right) M$ (recall $M$ is a linear space). Since $K / k$ is of finite type, it follows that $K \otimes_{k} K$ is noetherian; by assumption it is also integral, hence by Krull's theorem describing the intersection of powers of an ideal 4, Proposition III.3.5] $\left(\bigcap_{n \geq 1} I_{K}^{n}\right) M=0$ so $d_{K / k, M}$ is injective.

A simple example where the Taylor map $\mathrm{d}_{V}$ never is injective is provided by any nontrivial finite algebraic extension $K / k$, with $V=K=M$; since $\mathcal{P}_{K / k}^{n}=K$, the Taylor map will never be injective, when $|V| \geq 2$.

Proposition 2.2.3 implies easily that there exists an integer $N$ such that if $n \geq N$, then the map

$$
\mathrm{d}_{V}^{n}: \mathcal{V}_{X} \rightarrow \mathcal{P}_{X / k}^{n}(M)
$$

is injective in this situation. We will refine this result by investigating conditions that may be put on $V$ instead of $X / k$.

Proposition 2.2.4. Let $R$ be a local noetherian integral $k$-algebra such that its residue field $l$ is a finitely generated extension of $k$, let $M$ be a free $R$-module and let $V$ be finite-dimensional $k$-subspace of $M$. Let $k^{r}$ be the algebraic closure of $k$ in the quotient field $K=K(R)$ of $R$ and $k^{l}$ its algebraic closure in l. Let $R^{r}=k^{r} R \subset K$ be the $k^{r}$-algebra generated by $k^{r}$ and $R$, and $M^{r}=k^{r} M$ the $k^{r}$-space generated by $M$ in $K \otimes_{R} M$. The following are equivalent:

(1) There is an integer $N$ such that

$$
l \otimes_{k} V \rightarrow l \otimes_{R} \mathcal{P}_{R / k}^{n} \otimes_{R} M
$$

is injective when $n \geq N$.

(2) The map

is injective.

$$
l \otimes_{k} V \rightarrow l \otimes_{R} \mathcal{P}_{R / k}^{\infty} \otimes_{R} M
$$

(3) The map

$$
k^{l} \otimes_{k} V \rightarrow \frac{k^{l} \otimes_{k} k^{r}}{J_{0}} \otimes_{k^{r}} M^{r}
$$

is injective, where

$$
J_{0}=\left\{y \in k^{l} \otimes_{k} k^{r} \subset k^{l} \otimes_{k} R^{r} \mid \exists m \in I, \text { s.t. }(1+m) y=0\right\}
$$

and

$$
I=\operatorname{Ker}\left(l \otimes_{k} R \rightarrow l\right) .
$$

We have

$$
J_{2} \subset J_{0} \subset J_{1},
$$

where $J_{1}=\operatorname{Ker}\left(k^{l} \otimes_{k} k^{r} \rightarrow l \otimes_{R} R^{r}\right)$ and $J_{2}$ is the ideal in $k^{l} \otimes_{k} k^{r}$ generated by $\operatorname{Ker}\left(k^{l} \otimes_{k} \hat{k} \rightarrow k^{l}\right)$, where $\hat{k}$ is the algebraic closure of $k$ in $R$. 
Proof. It suffices to prove this when $M=R$. There is an injective map $l \otimes_{R} \mathcal{P}_{R / k}^{\infty} \rightarrow$ $\varliminf_{n} l \otimes_{R} \mathcal{P}_{R / k}^{n}$, so (2) holds if and only if the map $l \otimes_{k} V \rightarrow \varliminf_{n} l \otimes_{R} \mathcal{P}_{R / k}^{n}$ is injective. This is a reformulation of (1), since $V$ is finite-dimensional. Furthermore, we have a short exact sequence $0 \rightarrow I^{n} \rightarrow l \otimes_{k} R \rightarrow l \otimes_{R} \mathcal{P}_{R / k}^{n} \rightarrow 0$ (where

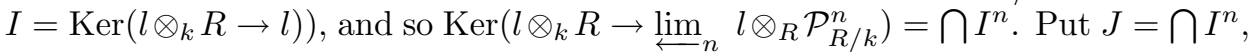
so (2) holds if and only if the map $l \otimes_{k} V \rightarrow \frac{l}{\frac{l n}{J}} R$ is injective. Since $l / k$ is of finite type, the ring $l \otimes_{k} R$ is noetherian by Hilbert's basis theorem. Furthermore, by Krull's theorem the ideal $J$ consists of the elements $s \in l \otimes_{k} R$ such that there exists $x \in I$ satisfying $(1+x) s=0$. Consider the inclusions

$$
S:=l \otimes_{k} R \subset \bar{S}=l \otimes_{k} R^{r} \subset S_{K}=l \otimes_{k} K .
$$

We have $R \subset S$ and $R \cap J=0$, since elements in $R$ are not zero-divisors in $S$. Hence $\bar{J}:=\bar{S} J$ and $J_{K}:=S_{K} J$ are proper ideals. Furthermore, $S \cap J_{K}=J$; for if $y / f=s \in S \cap J_{K}$, there exists $1+x \in 1+I$, such that $(1+x) y=f(1+x) s=0$, hence $(1+x) s=0$ and so $s \in J$. This means that there are maps

$$
S / J \rightarrow \bar{S} / \bar{J} \rightarrow S_{K} / J_{K}
$$

such that the composition is injective. Hence (2) is equivalent to the injectivity of

$$
l \otimes_{k} V \rightarrow \frac{l \otimes_{k} K}{J_{K}}
$$

Now $S_{K}=l \otimes_{k^{l}}\left(k^{l} \otimes_{k} k^{r}\right) \otimes_{k^{r}} K$. If $P$ is a prime ideal of $S_{0}:=k^{l} \otimes_{k} k^{r}$, then $S_{K} / S_{K} P=l \otimes_{\tilde{l}}\left(S_{0} / P\right) \otimes_{k^{r}} K$ is integral (Lemma 2.2.2); hence $S_{K} P$ is a prime ideal. According to [4, IV, Prop. 11], if $0=\bigcap P_{i}$ in $S_{0}$, we then also have $0=\bigcap S_{K} P_{i}\left(S_{0}\right.$ and $S$ are reduced); this implies that the minimal primes of $S_{0}$ and $S_{K}$ correspond, and more generally $A=S_{K}\left(A \cap S_{0}\right)$ is true for all radical ideals. Since all ideals in $S_{K}$ (a direct sum of fields) are radical, this is true for all ideals. Define $J_{0}:=S_{0} \cap J_{K}$; then the equivalence of (2) with (3) is immediate: $S_{K} / J_{K}=l \otimes_{k^{l}}\left(S_{0} / J_{0}\right) \otimes_{k^{r}} K$, and the map $l \otimes_{k} V \rightarrow \frac{l \otimes_{k} K}{J_{K}}$ is a flat extension of the map $k^{l} \otimes_{k} V \rightarrow\left(S_{0} / J_{0}\right) \otimes_{k^{r}} K$. The characterisation of $J_{0}$ follows from the fact that $J_{K}=\left\{y \in S_{K} \mid \exists m \in I\right.$, s.t. $\left.(1+m) y=0\right\}$.

To estimate $J_{0}$, note first that $A:=\operatorname{Ker}\left(k^{l} \otimes_{k} \hat{k} \rightarrow k^{l}\right) \subset I$, that $A^{n}=A$, since $k^{l} \otimes_{k} \hat{k}$ is a direct sum of fields, and that hence $J_{2}=S_{0} A \subset J_{K}$, implying that $J_{2} \subset J_{0}$. Then put $\bar{I}=\bar{S} I=\operatorname{Ker}\left(l \otimes_{k} k^{r} R \rightarrow l \otimes_{R} R^{r}\right)$. We have an inclusion

$$
\bar{J}=\bar{S} J \subset B:=\bigcap \bar{S} I^{n}=\bigcap \bar{I}^{n} .
$$

Since $k^{l} \otimes_{k} k^{r} \subset l \otimes_{k} R^{r}=\bar{S}$, we can define $J_{1}=\operatorname{Ker}\left(k^{l} \otimes_{k} k^{r} \rightarrow l \otimes_{R} R^{r}\right) \subset \bar{S}$. Then $J_{1}=S_{0} \cap \bar{I} \subset \bar{I}$, and hence, as before, $J_{1} \subset B=\bigcap \bar{I}^{n}$. We assert that $J_{K} \subset S_{K} \cap B=S_{K} J_{1}$. The inclusion is immediate, and the description of $B$ follows, in the same way as above, since $J_{1}=S_{0} \cap\left(\cap \bar{I}^{n}\right)$, and since $\bar{S} \cap S_{K} B=B$, by the characterisation $B=\left\{y \in S_{K} \mid \exists m \in \bar{I}\right.$, s.t. $\left.(1+m) y=0\right\}$ (cf. the similar argument above).

By reduction to $\mathcal{O}_{x}=R$ and $M=M_{x}$, we get the following analysis of the behaviour of the Taylor map at a point $x$.

Theorem 2.2.5. Assume $X$ is an integral variety, $M$ a locally free $\mathcal{O}_{X}$-module and $V$ a k-subspace of $\Gamma(X, M)$, and let $x$ be a point in $X$. Denote the algebraic 
closure of $k$ in the function field $k(X)$ by $k^{r}$, and the $k^{r}$-module generated by $M_{x}$ in $k(X) \otimes_{\mathcal{O}_{x}} M_{x}$ by $M_{x}^{r}$. The following are equivalent:

(1) There is an integer $N$ such that the map

$$
k_{x} \otimes_{k} V \rightarrow k_{x} \otimes_{\mathcal{O}_{x}} \mathcal{P}_{X / k}^{n}(M)_{x}
$$

is injective when $n \geq N$.

(2) The map

$$
k_{x}^{l} \otimes_{k} V \rightarrow\left(\left(k_{x}^{l} \otimes_{k} k^{r}\right) / J_{0}\right) \otimes_{k^{r}} M_{x}^{r}
$$

is injective, where $k_{x}^{l}$ is the algebraic closure of $k$ in $k_{x}$ and $J_{0}$ is the ideal defined in the following way:

$$
J_{0}=\left\{y \in k_{x}^{l} \otimes_{k} k^{r} \mid \text { there is } m \in I \text { such that }(1+m) y=0\right\} .
$$

Definition 2.2.6. If the equivalent conditions in Proposition 2.2.4 hold, we say that $V$ is a separated subspace of $M$. If the equivalent conditions in Theorem 2.2.5 hold at $x$, we say that $V$ is separated at $x$, and if these conditions hold at each point, we say that $V$ is separated (on $X$ ).

Corollary 2.2.7. Let $X / k, V$ and $M$ be as in Theorem 2.2.5.

(1) If $X$ is geometrically integral, then $V$ is separated at all points.

(2) If $x$ is a rational point, then $V$ is separated at $x$.

(3) If $x$ is a normal point, then $V$ is separated if and only if the map

$$
k^{r} \otimes_{k} V \rightarrow M_{x}^{r}
$$

is injective.

(4) If the map $k^{r} \otimes_{k} V \rightarrow M_{x}^{r}$ is injective, then $V$ is separated at $x$.

Proof. (1) If $X$ is geometrically integral, then $k^{r}=k$ (Proposition 2.2.2) so $k_{x}^{l} \otimes_{k}$ $k^{r}=k_{x}^{l}$ and since $J_{0}$ is proper, it is hence the zero-ideal. Thus the map in part (2) of Theorem 2.2.5 is just the inclusion $V \rightarrow M_{x}$, tensored by the flat $k$-module $k_{x}^{l}$.

(2) If $x$ is a rational point $k_{x}^{l}=l$ and hence again $J_{0}=0$, and the map becomes the inclusion.

(3) If $x$ is normal, then $\overline{\mathcal{O}}_{x}=\mathcal{O}_{x}$ and $\hat{k}=k^{r}$, and hence $J_{1}=J_{2}=J_{0}$, and so $\frac{k_{x}^{l} \otimes_{k} k^{r}}{J_{0}}=k_{x}^{l}$. This means that the sequence of the proposition becomes $k_{x}^{l} \otimes_{k} V \rightarrow k_{x}^{l} \otimes_{k^{r}} k^{r} M_{x}$. This is by flatness injective if and only if the map $k^{r} \otimes_{k} V \rightarrow M^{r}$ is injective.

(4) From the assumption, it follows that the map

$$
\frac{k_{x}^{l} \otimes_{k} k^{r}}{J_{0}} \otimes_{k^{r}} k^{r} \otimes_{k} V \rightarrow \frac{k_{x}^{l} \otimes_{k} k^{r}}{J_{0}} \otimes_{k^{r}} M_{x}^{r}
$$

is injective. However, the first vector space is isomorphic to $\frac{k_{x}^{l} \otimes_{k} k^{r}}{J_{0}} \otimes_{k} V$, and contains $k_{x}^{l} \otimes_{k} V$. Hence (4) follows from Proposition 2.2.4

Remark 2.2.8. The following are equivalent:

(1) $V$ is a separated subspace of $M_{x}$ at each associated point $x$;

(2) there exist an integer $N$ such that the Taylor map $\mathrm{d}_{V}^{n}: \mathcal{V} \rightarrow \mathcal{P}_{X / k}^{n}$ is injective when $n \geq N$. 
Definition 2.2.9. Let $n_{i n j}(x)=n_{i n j}(x, V)$ be the smallest integer such that $\mathrm{d}_{V}^{n}(x)$ is injective (injectivity order of $V$ at $x$ ). Define also

$$
\begin{gathered}
n_{i n j}=n_{i n j}(V)=\sup \left\{n_{i n j}(x) \mid x \in X\right\}, \\
N_{i n j}=N_{i n j}(V)=\inf \left\{n \mid \mathrm{d}_{V}^{n} \text { is injective }\right\} .
\end{gathered}
$$

The integer $n_{i n j}$ is the injectivity order of $V$ and $N_{i n j}$ the generic injectivity order.

Proposition 2.2.10 $(X / k$ is a smooth scheme locally of finite type). Let $M$ be a locally free $\mathcal{O}_{X}$-module and $V$ a subspace of $\Gamma(X, M)$. Then the function $x \mapsto$ $n_{\text {inj }}(x)$ is upper semi-continuous. In particular, if $x$ is a specialisation of a point $y$, then $n_{i n j}(x) \geq n_{\text {inj }}(y)$, and if $X$ is irreducible, then for any point $x$ we have $n_{\text {inj }}(x) \geq N_{\text {inj }}$.

We will see in Proposition 3.1.1 that $N_{i n j} \leq \operatorname{dim} V-1$.

Proof. Let $x$ be a specialisation of the point $y$, and let $n \geq n_{i n j}(x)$. By Proposition 2.2.1. (1) $\Rightarrow(2)$, the map $\mathrm{d}_{V, x}^{n}$ is split injective, hence it is split injective in some neighbourhood $\Omega$ of $x$; since $y$ specialises to $x$ we have $y \in \Omega$, implying that the map $\mathrm{d}_{V, y}^{n}$ is split injective, hence by Proposition 2.2.1 (2) $\Rightarrow(1), n \geq n_{i n j}(y)$. It is also clear that sets of the form $\left\{x \in X \mid n_{i n j}(x)<i\right\}$ are open for each integer $i$. Since $\mathcal{V}_{X}$ is free, $N_{i n j}=\sup \left\{n_{i n j}(\eta) \mid \eta\right.$ is an associated point of $\left.X\right\}$, so, in particular, $n_{i n j}(x) \geq N_{i n j}, x \in X$, when there is only one associated point.

By Proposition 2.2.10 the function $x \mapsto n_{i n j}(x)$ is upper semi-continuous when $X$ is a regular variety, hence $n \geq n_{i n j}$ if $\mathrm{d}_{V}^{n}(x): k_{x} \otimes_{k} V \rightarrow k_{x} \otimes_{\mathcal{O}_{x}} \mathcal{P}_{X / k}^{n}(M)_{x}$ is injective at each closed point $x$. Actually, if $X / k$ is any scheme locally of finite type with trivial Jacobson radical it follows from the Nullstellensatz that $n \geq n_{i n j}$ if $\mathrm{d}_{V}^{n}(x): k_{x} \otimes_{k} V \rightarrow k_{x} \otimes_{\mathcal{O}_{x}} \mathcal{P}_{X / k}^{n}(M)_{x}$ is injective at each closed point $x$.

Theorem 2.2.11. Let $X / k$ be a reduced scheme locally of finite type, $M$ a locally free $\mathcal{O}_{X}$-module, and $V$ be a finite-dimensional separated $k$-subspace of $\Gamma(X, M)$ (e.g., $k$ is algebraically closed and $V$ injects to each stalk $M_{x}$ ). Then the injectivity order is finite, $n_{i n j}(V)<\infty$.

Proof. a) By Proposition 2.2.10, sets of the form $U_{k}=\left\{x \mid n_{i n j}(x)<k\right\}$ are open. By the noetherianness, this implies that there is an integer $N$ such that $k \geq N$ implies that $U_{k}=U_{N}$. Since $V$ is separated at each point in $X$, this implies that $U_{N}=X$. This proves the theorem when $X / k$ is regular.

b) Assuming $X / k$ is not regular there exists a nonempty closed subset $X_{s} \subset X$ such that $\operatorname{dim} X_{s}<\operatorname{dim} X$ and $X_{0}=X \backslash X_{s}$ is regular [15, III.10.5]. Provide $X_{s}$ with its reduced scheme structure; then $X_{s} / k$ again is a scheme of finite type. Let $j: X_{s} \rightarrow X$ be the associated closed immersion. To prove the theorem we will use induction over $\operatorname{dim} X$. By a), $n_{i n j}^{0}:=\sup \left\{n_{i n j}(x) \mid x \in X_{0}\right\}<\infty$. If $\operatorname{dim} X=1$, its singular locus $X_{s}$ is a union of finitely many points $x_{1}, \ldots, x_{r}$ so $n_{i n j}=\max \left\{n_{i n j}^{0}, n_{i n j}\left(x_{1}\right), \ldots, n_{i n j}\left(x_{r}\right)\right\}<\infty$. We will extend this argument.

Let $\xi$ be an associated point of $X_{s} \subset X$ and consider the following commutative diagram

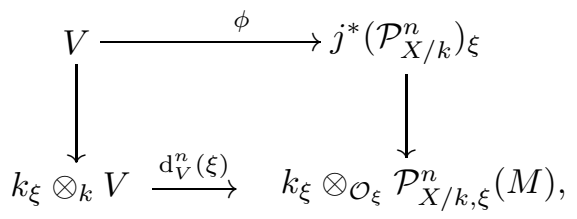


where $\phi: V \rightarrow j^{*}\left(\mathcal{P}_{X / k}^{n}\right)$ is the canonical map. By Definition 2.2.6, there exists an integer $n_{i n j}(\xi)$ such that the map $\mathrm{d}_{V}^{n}(\xi): k_{\xi} \otimes_{k} V \rightarrow k_{\xi} \otimes_{\mathcal{O}_{\xi}} \mathcal{P}_{X / k, \xi}^{n}(M)$ is injective when $n \geq n_{i n j}(\xi)$, hence $\phi$ is injective when $n \geq n_{2}:=\max \left\{n_{\text {inj }}(\xi) \mid \xi\right.$ is an associated prime of $\left.X_{s}\right\}$.

This allows us to use the induction hypothesis. We conclude that there is an integer $n_{1}$ such that the induced map $\gamma: k_{x} \otimes_{k} V \rightarrow k_{x} \otimes_{\mathcal{O}_{X_{s}, x}} \mathcal{P}_{X_{s} / k, x}^{n_{1}}\left(j^{*} \mathcal{P}_{X / k}^{n_{2}}(M)\right)$ is injective for each $x \in X_{s}$. By [14, Prop. 16.4.20, 16.7.9] we have a surjective map

$$
\alpha: j^{*}\left(\mathcal{P}_{X / k}^{n_{1}}\left(\mathcal{P}_{X / k}^{n_{2}}(M)\right)\right) \rightarrow \mathcal{P}_{X_{s} / k}^{n_{1}}\left(j^{*} \mathcal{P}_{X / k}^{n_{2}}(M)\right)
$$

and by [14, Lemme 16.8.9.1, 16.7.9] a map

$$
\beta: \mathcal{P}_{X / k}^{n_{1}+n_{2}}(M) \rightarrow \mathcal{P}_{X / k}^{n_{1}}\left(\mathcal{P}_{X / k}^{n_{2}}(M)\right)
$$

Since, by functoriality, $\alpha(x) \circ \beta(x) \circ \mathrm{d}_{X, V}^{n_{1}+n}(x)=\gamma$ we obtain that $\mathrm{d}_{X, V}^{n_{1}+n_{2}}(x)$ is injective if $x \in X_{s}$. Hence $\mathrm{d}_{V}^{n}(x)$ is injective for each $x \in X$ if $n \geq \max \left\{n_{i n j}^{0}, n_{1}+\right.$ $\left.n_{2}\right\}$.

Remark 2.2.12. The assertion $n_{i n j}(V)<\infty$ is proven in 23 for smooth schemes $X / S$ with geometrically irreducible fibres. In the language of [21] the corresponding assertion is that $V$ is a "regular" subspace of $M$. Since they only consider varieties $X$ that are open subsets of some $\mathbf{R}^{n}$, which clearly are geometrically integral, arbitrary finite-dimensional subspaces $V$ of functions will be separable. In the analytic case $X_{0}$ may not be relatively quasi-compact and $n_{i n j}(V)$ may thus be infinite for different reasons than in the algebraic situation.

2.3. The surjectivity of the Taylor map. Surjectivity properties of the Taylor map will play a role in extending differential operators.

Definition 2.3.1. Let $n_{\text {surj }}(x)$ be the largest integer such that $n \leq n_{\text {surj }}(x)$ implies that $\mathrm{d}_{V}^{n}(x)$ is surjective ( $\operatorname{so}_{x}^{n}=0$ ). The integer $n_{\text {surj }}(x)$ is the jet order of $V$ at $x$. Define $n_{\text {surj }}=\inf \left\{n_{\text {surj }}(x): x \in X\right\}$. Define also $n_{\text {surj }}^{1}$ to be the largest integer such that $\operatorname{codim} \operatorname{supp} \mathcal{C}^{n} \geq 2$ when $n \leq n_{\text {surj }}^{1}$.

From (2.2) it is evident that if $x$ is a rational point, then

$$
n_{\text {surj }}(x) \leq \max \left\{n \mid \operatorname{dim}_{k} M_{x} / \mathfrak{m}_{x}^{n+1} M_{x} \leq \operatorname{dim}_{k} V\right\} .
$$

The following well-known lemma illustrates the meaning of $n_{\text {surj }}(x) \geq 1$. As mentioned in the introduction and as will be described later, every linear system $V \subset \Gamma(X, M)$ corresponds to a rational map $X \rightarrow \mathbf{P}^{n}$, for some $n$. In the lemma the conditions for this map to be a closed embedding is related to $n_{\text {surj }}$.

Lemma 2.3.2 ( $k$ algebraically closed). Assume that $V \subset \Gamma(X, M)$ is a linear system on a nonsingular projective variety $X$, where $M$ is an invertible sheaf. Then

(1) $n_{\text {surj }} \geq 0$ if and only if $V$ generates $M$.

(2) If $V$ defines a closed embedding, e.g., $V=\Gamma(X, M)$ and $M$ is very ample, then for each point $x$ in $X$ we have $n_{\text {surj }}(x) \geq 1$.

(3) If $n_{\text {surj }}(x) \geq 1$ and in addition $V$ separates points, i.e., the canonical map $V \rightarrow k_{x} \otimes_{\mathcal{O}_{x}} M_{x} \oplus k_{y} \otimes_{\mathcal{O}_{y}} M_{y}$ is surjective when $x$ and $y$ are different closed points in $X$, then $V$ defines a closed embedding. 
For the proof, consult [15, Prop. II.7.3].

More generally $n_{\text {surj }}(x) \geq n$ is implied by the linear system being $n$-jet ample (cf. [5]). In the definition of the first concept, in contrast to the second, only infinitesimal subschemes with support in a point are considered. Hence it is in general weaker.

Proposition 2.3.3. Let $M$ be a locally free $\mathcal{O}_{X}$-module. Then $n_{\text {sur } j} \leq n_{\text {sur } j}^{1} \leq$ $N_{i n j}$.

Proof. The first inequality is obvious. Assuming $N_{i n j}<n \leq n_{s u r j}^{1}$, then $\mathrm{d}_{V}^{n}$ is an injective map that is surjective at points of height 1 in the locus if nonsingular points $X_{0}$. As $X_{0}$ is a regular variety $\mathcal{P}_{X_{0} / k}^{n}(M)$ is locally free so its depth at points of height $\geq 2$ is at least 2 ; therefore $\mathrm{d}_{V}^{n}$ is an isomorphism. But since $n>N_{i n j}$, the rank of $\mathcal{P}_{X_{0} / k}^{n}(M)$ is greater than the rank of $\mathcal{V}_{X_{0}}$, which gives a contradiction. Therefore $n_{\text {surj }}^{1} \leq N_{\text {inj }}$.

We can easily see when equality holds in the preceding proposition, using a characterisation of projective spaces in terms of osculating spaces by Fulton et al. in 9. The result shows that $\mathbf{P}^{n}$ is characterised in terms of extendibility of differential operators (see Theorem 4.2.1), a fact that is perhaps not surprising in view of Mori's characterisation of projective space by the ampleness of its tangent bundle (this result is the hard part of [9]).

Proposition 2.3.4 ( $k$ algebraically closed). Assume that $V=\Gamma(X, M)$ is a very ample linear system on a nonsingular projective variety $X$ of dimension $n$, where $M$ is an invertible sheaf. The following are equivalent:

(1) $n_{\text {inj }}=n_{\text {surj }}$;

(2) $N_{\text {inj }}=n_{\text {surj }}^{1}$;

(3) $X=\mathbf{P}^{n}$ and $M=\mathcal{O}(k)$, for some integer $k \geq 1$.

Proof. The first statement trivially implies the second, in view of Propositions 2.3 .3 and 2.2.10. The second statement implies that $\mathrm{d}_{V}^{k}$, for $k=N_{i n j}$ is injective and surjective at all points of height 1 , hence the dual is an isomorphism. Then the proof of the Theorem in [9, p. 206], shows that the third statement is true. The final implication of the proof is deferred until Section 5.1.

\section{Differential operators PRESERVing $V$}

3.1. Weierstrass points. Applying $\operatorname{Hom}_{\mathcal{O}_{X}}(\cdot, M)$ to the Taylor sequence (2.1) we get a map

$$
\mathcal{D}_{X}^{n}(M) \stackrel{W^{n}}{\longrightarrow} \operatorname{Hom}_{\mathcal{O}_{X}}\left(\mathcal{V}_{X}, M\right) \cong \operatorname{Hom}_{k}(V, M) .
$$

A (local) differential operator $P \in \mathcal{D}_{X}^{n}(M):=\operatorname{Hom}_{\mathcal{O}_{X}}\left(\mathcal{P}_{X / k}^{n}(M), M\right)$, induces a (local) map $\operatorname{Hom}_{k}(M, M)$, or an action on $M$, by $m \mapsto P \cdot m:=P\left(d^{n}(m)\right)$, where $d^{n}: M \rightarrow \mathcal{P}_{X / k}^{n}(M)$ is the canonical map, described in 2.1. Then $W^{n}$ takes $P$ to the map $V \ni v \mapsto P \cdot v$, and we may describe the kernel of $W^{n}$ as the annihilator $\operatorname{Ann}^{n}(V)=\left\{P \in \mathcal{D}_{X}^{n}(M) \mid P \cdot V=0\right\}$. It is hence clear that $V$ defines an $\mathcal{O}_{X^{-}}$ coherent left $\mathcal{D}_{X}(M)$-module

$$
\mathcal{D}_{X}(M) / \operatorname{Ann}(V) \cong \bigcup_{n=0}^{\infty} W^{n}\left(\mathcal{D}^{n}(M)\right) \subset \operatorname{Hom}_{\mathcal{O}_{X}}\left(\mathcal{V}_{X}, M\right),
$$


where $\operatorname{Ann}(V)=\bigcup_{n=0}^{\infty} \operatorname{Ann}(V)$. Note that its rank is less than or equal to $\operatorname{dim} V$. $\operatorname{rank} M$. We will first use this to give the upper bound on $N_{i n j}(V)$, referred to before, when $V$ is separated at each associated point. This proposition is essentially proved in 23]; we have extended the result slightly and our setup perhaps gives a more "conceptual" proof.

Proposition 3.1.1 $(X / k$ is a reduced scheme of characteristic 0$)$. Assume that the map $\mathrm{d}_{V}: \mathcal{V}_{X} \rightarrow \mathcal{P}_{X / k}^{\infty}$ is injective (see Remark 2.2.8). Then $\mathrm{d}_{V}^{n}$ is injective when $n \geq \operatorname{dim} V-1$, i.e., $N_{i n j}(V) \leq \operatorname{dim} V-1$. Also, the map $W_{x}^{n}$ is surjective at each associated point $x$ when $n \geq \operatorname{dim} V-1$.

We need a well-known fact.

Lemma 3.1.2. If $K / k$ is a finitely generated field extension of characteristic 0 , then the ring of differential operators $\mathcal{D}_{K / k}$ is generated by its subspace of first order differential operators.

By the references in the proof of Lemma 2.1.1, $K / k$ is differentially smooth (char. $0)$. Then the proof that $\mathcal{D}_{K / k}$ is generated by $\mathcal{D}_{K / k}^{1}$ follows immediately from [14, Th. 16.11.2]. (One may also notice that $\mathcal{D}_{K / k}(M) \cong \mathcal{D}_{K / k} \otimes_{K} \operatorname{End}_{K}(M)$ is a matrix algebra of the ring $\mathcal{D}_{K / k}$, hence it is generated by matrices of differential operators in $\mathcal{D}_{K / k}^{1}$.)

Proof of Proposition 3.1.1. The map $\mathrm{d}_{V}^{n}$ is injective if $\mathrm{d}_{V, x}^{n}$ is injective for each associated point $x$, and then $\mathrm{d}_{V, x}^{n}$ is injective if and only if $W_{x}^{n}$ is surjective since $\mathcal{O}_{x}$ is a field. It suffices therefore to prove the following: Let $K / k$ be a field extension and $M$ a finite-dimensional $K$-linear space with a finite-dimensional $k$-linear subspace $V \subset M$. Then if the map $W: \mathcal{D}_{K / k}(M) \rightarrow \operatorname{Hom}_{k}(V, M), P \mapsto(v \mapsto P \cdot v)$ is surjective, it follows that the map $\mathcal{D}_{K / k}^{n}(M) \rightarrow \operatorname{Hom}_{k}(V, M)$ is surjective when $n \geq \operatorname{dim}_{k} V-1$. Clearly, it suffices to prove this when $M=K$ and $V \subset K$. Moreover, if $W$ is surjective, since $\operatorname{dim}_{k} V<\infty$ there exists a subfield $K_{1} \subset K$ that is finitely generated over $k$ such that $\mathcal{D}_{K_{1} / k} \rightarrow \operatorname{Hom}_{k}\left(V, K_{1}\right)$ is surjective. Then if $\mathcal{D}_{K_{1} / k}^{n} \rightarrow \operatorname{Hom}_{k}\left(V, K_{1}\right)$ is surjective, it follows that $\mathcal{D}_{K / k}^{n} \rightarrow \operatorname{Hom}_{k}(V, K)$ is surjective, since any element in $\mathcal{D}_{K_{1} / k}^{n}$ can be lifted to an element in $\mathcal{D}_{K / k}^{n}$. We can therefore also assume that $K$ is finitely generated over $k$.

The space $\operatorname{Hom}_{k}(V, K)$ is a module over $\mathcal{D}_{K / k}$, where a differential operator $P$ acts on $\phi: V \rightarrow K$ by $(P \cdot \phi)(v)=P(\phi(v))$. One then has $\operatorname{Im} W^{n}=\mathcal{D}_{K / k}^{n} \operatorname{Im} W^{0}$ and $\operatorname{dim}_{K} \operatorname{Im} W^{0}=1$. Assume $\operatorname{Im} W^{n}=\operatorname{Im} W^{n+1}$, i.e., $\mathcal{D}_{K / k}^{n+1} \operatorname{Im} W^{0}=\mathcal{D}_{K / k}^{n} \operatorname{Im} W^{0}$. Hence by Lemma 3.1 .2 ,

$$
\mathcal{D}_{K / k}^{n} \operatorname{Im} W^{0}=\mathcal{D}_{K / k}^{1} \mathcal{D}_{K / k}^{n} \operatorname{Im} W^{0},
$$

and hence also that $\mathcal{D}_{K / k}^{n} \operatorname{Im} W^{0}=\mathcal{D}_{K / k} \operatorname{Im} W^{0}$. By assumption $\operatorname{Im} W=$ $\mathcal{D}_{K / k} \operatorname{Im} W^{0}=\operatorname{Hom}_{k}(V, K)$, and $\operatorname{dim}_{K} \operatorname{Hom}_{k}(V, K)=\operatorname{dim}_{k} V<\infty$; hence there exists a smallest integer $n_{0}$ such that $\operatorname{Im} W^{n}=\operatorname{Hom}_{k}(V, K)$ when $n \geq n_{0}$. We have

$$
\operatorname{Im} W^{0} \subsetneq \operatorname{Im} W^{1} \subsetneq \cdots \subsetneq \operatorname{Im} W^{n_{0}}=H o m_{k}(V, K),
$$

so the dimensions increase at each step. Hence $n_{0}+1 \leq \operatorname{dim}_{K} \operatorname{Hom}_{k}(V, K)=$ $\operatorname{dim}_{k} V$, i.e., $n_{0} \leq \operatorname{dim}_{k} V-1$. 
A gap for $V$ at a point $x$ in $X$ is an integer $i$ such that $\operatorname{rkd}_{V}^{i}(x)>\operatorname{rkd}_{V}^{i-1}(x)$, and the gap sequence of $V$ at $x$ is the set of gap integers. We see from the proof of Proposition 3.1.1 that the gap sequence at an associated point $\xi$ of a separated subspace $V \subset \Gamma(X, M)$ is $1,2, \ldots, n_{i n j}(\xi)$ where $n_{i n j}(\xi) \leq \operatorname{dim} V-1$. (If tr. $\operatorname{deg}_{k} K=$ 1, then $N_{i n j}=\operatorname{dim} V-1$.) Assuming $X$ is irreducible, one says that a point $x$ in $X$ is aWeierstrass point for $V$ if its gap sequence is different from the generic gap sequence. From Proposition 2.2.10 it follows that a point $x$ on a nonsingular variety $X / k$ is a Weierstrass point if and only if $n_{i n j}(x)>N_{i n j}$.

For each integer $j \geq 0$ we have a short exact sequence

$$
0 \rightarrow \mathcal{V} \stackrel{\mathrm{d}_{V}^{N_{i n j}+j}}{\longrightarrow} \mathcal{P}_{X / k}^{N_{i n j}+j}(M) \rightarrow \mathcal{C}^{N_{i n j}+j} \rightarrow 0
$$

and can define the subsets

$$
W_{j}:=W_{j}(V)=\left\{x \in X \mid n_{i n j}(x)>N_{i n j}+j\right\} ;
$$

put also $W=W_{0}(V)$. The closed set $W(V)$ (by the semi-continuity of $n_{\text {inj }}(x)$ ) can be regarded as a set of Weierstrass points on $X$ for $V$, and $W_{j}(V)$ is its subset of Weierstrass points of order $j$. We have

$$
\emptyset=W_{n_{i n j}-N_{i n j}} \subseteq \cdots \subseteq W_{j} \subseteq \cdots \subseteq W_{1} \subseteq W .
$$

We can express these sets as supports of a coherent $\mathcal{O}_{x}$-module.

Proposition 3.1.3 $(X / k$ is a regular variety). We have

$$
W_{j}=\operatorname{supp} E x t_{\mathcal{O}_{X}}^{1}\left(\mathcal{C}^{N_{i n j}+j}, \mathcal{O}_{X}\right)
$$

so in particular $W_{j}, j=0,1, \ldots, n_{i n j}-N_{i n j}+1$, are proper closed subsets.

Proof. $\operatorname{supp} E x t_{\mathcal{O}_{X}}^{1}\left(\mathcal{C}^{N_{i n j}+j}, \mathcal{O}_{X}\right) \subseteq W_{j}(V)$ : Assume $x \notin W_{j}(V)$. By Proposition 2.2.1, (1) $\Rightarrow(2)$, the Taylor sequence (2.1), with $n=N_{i n j}$ and $\mathcal{K}^{N_{i n j}}=0$, is split exact since $\mathcal{P}_{X / k}^{N_{i n j}+j}(M)$ is locally free. Therefore $\mathcal{C}_{x}^{N_{i n j}+j}$ is free over $\mathcal{O}_{x}$; hence, $\mathcal{C}^{N_{i n j}+j}$ being coherent, $\operatorname{Ext}_{\mathcal{O}_{X}}^{1}\left(\mathcal{C}^{N_{i n j}+j}, \mathcal{O}_{X}\right)_{x}=\operatorname{Ext}_{\mathcal{O}_{x}}^{1}\left(\mathcal{C}_{x}^{N_{i n j}+j}, \mathcal{O}_{x}\right)=0$, so $x \notin \operatorname{supp} E x t_{\mathcal{O}_{X}}^{1}\left(\mathcal{C}^{N_{i n j}+j}, \mathcal{O}_{X}\right)$.

$W_{k}(V) \subseteq \operatorname{supp} E x t_{\mathcal{O}_{X}}^{1}\left(\mathcal{C}^{N_{i n j}+j}, \mathcal{O}_{X}\right):$ If $x \notin \operatorname{supp} E x t_{\mathcal{O}_{X}}^{1}\left(\mathcal{C}^{N_{i n j}+j}, \mathcal{O}_{X}\right)$, then (2.1), localised at $x$, is split exact; hence by Proposition 2.2.1. $(2) \Rightarrow(1), n_{i n j}(x)=$ $N_{i n j}+j$; hence $x \notin W_{j}(V)$.

That supp $\operatorname{Ext}_{\mathcal{O}_{X}}^{1}\left(\mathcal{C}^{N_{i n j}+j}, \mathcal{O}_{X}\right)$ is a closed proper subset of $X$ is clear since $\mathcal{C}^{N_{i n j}+j}$ is coherent.

By Proposition 3.1.3, a natural scheme structure on $W_{j}(V)$ is given by the coherent ideal $\operatorname{Ann}_{\mathcal{O}_{X}} \operatorname{Ext}_{\mathcal{O}_{X}}^{1}\left(\mathcal{C}^{N_{i n j}+j}, \mathcal{O}_{X}\right)$.

Remark 3.1.4. Another way to say that $x \in W_{j}$ is that there exists a nonzero vector $v$ in $V$ such that $\mathcal{D}_{X / k}^{N_{i n j}+j}(M)_{x}(v) \subset \mathfrak{m}_{x} M_{x}$. If $V$ is a complete linear system of affine dimension $r$ for a very ample invertible sheaf $M$, with respect to an embedding in projective space $X \rightarrow \mathbf{P}^{r-1}\left(V^{*}\right)=\operatorname{Proj} \mathrm{S}_{k}(V)$, this means that there exists a hyperplane in $\mathbf{P}^{r-1}$ that has contact with $X$ of order $N_{i n j}+j$ at $x$ (an osculating plane).

Notice that the ideals $\operatorname{Ann}_{\mathcal{O}_{X}} \operatorname{Ext}_{\mathcal{O}_{X}}^{1}\left(\mathcal{C}^{N_{i n j}+j}, \mathcal{O}_{X}\right)$ define subschemes on any scheme $X / k$ locally of finite type (making $\mathcal{C}^{N_{i n j}+j}$ and hence the ideal coherent). Thus $\bar{W}_{j}:=\operatorname{supp} E x t_{\mathcal{O}_{X}}^{1}\left(\mathcal{C}^{N_{i n j}+j}, \mathcal{O}_{X}\right)$ is one candidate for Weierstrass sets of order 
$j$ for general schemes $X / k$, and also for general $\mathcal{O}_{X}$-modules $M$, although we cannot expect $\bar{W}_{j}=W_{j}$. Notice also that if $X / k$ is noetherian and reduced, $n_{i n j}<\infty$ (Theorem 2.2.11), so $W_{j}=\emptyset$ when $j \gg 1$, while it is not certain that $\bar{W}_{j}$ need be decreasing in the singular case.

Remark 3.1.5. In 23] Weierstrass points on nonsingular irreducible $X / k$ were defined using rank conditions on $\mathrm{d}_{V}^{i}(x)$, giving rise to a sequence of closed subsets $Z\left(w_{j}\right)=\left\{x \in X \mid \operatorname{rkd}_{V}^{i}(x)<\operatorname{rkd}_{V}^{i}(\xi)\right.$ for some $\left.i=0, \ldots, j-1\right\}$, where $\operatorname{rkd}_{V}^{i}(\xi)$ is the rank at the generic point $\xi$ in $X$. It is straightforward to see that $Z\left(w_{j}\right)$ equals $W(V)$ when $j \gg 1$, but for small $j$ the increasing filtration $\left\{Z\left(w_{j}\right)\right\}$ bears no natural connection to the decreasing filtration $\left\{W_{k}(V)\right\}$ of $W(V)$. In [27] Weierstrass points were defined differently. Let $X / k$ be a projective regular variety and $h$ the largest integer such that $\operatorname{rank} \mathcal{P}_{X / k}^{n} \leq \operatorname{dim}_{k} V$. Ogawa defines the sets

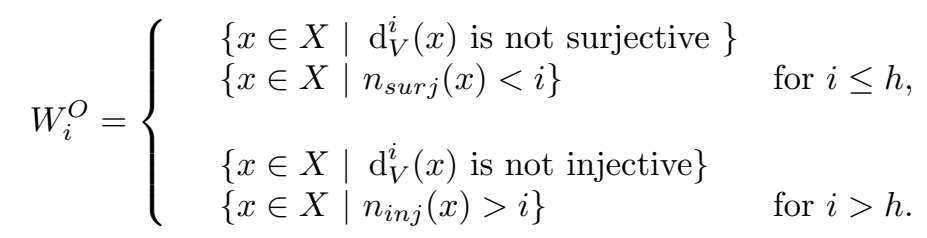

Where in the second line we have expressed these sets using the functions $n_{i n j}(x)$ and $n_{\text {surj }}(x)$. Ogawa does not prove that $W_{i}^{O}=\emptyset$ for sufficiently high $i$; this however was proven in 23. By Theorem 2.2.11 we get that $W_{i}^{O}=\emptyset$ for sufficiently high $i$ also when $X / k$ is any reduced scheme locally of finite type. As noticed in [23] the sets $W_{i}^{O}$ need not be proper subsets of $X$.

We are uncertain whether one can have $n_{i n j}(x)<N_{i n j}$ when $x$ is a singular point. This cannot however occur at rational points on a curve $X / k$ when $M$ is an invertible sheaf, for then $n \geq n_{i n j}(x)$ implies that $n \geq \operatorname{dim} V-1$, by (2.2), so $n \geq N_{i n j}$ (Proposition 3.1.1).

3.2. $M$ is simple as $\mathcal{D}_{X}(M)$-module when $X$ is regular. Let $V$ be a separated $k$-subspace of $\Gamma(X, M)$, so in particular there exists an integer $N_{i n j}$ such that $\mathrm{d}_{V}^{n}$ is injective when $n \geq N_{i n j}$. Applying $\operatorname{Hom}_{\mathcal{O}_{X}}(\cdot, M)$ to the Taylor sequence (2.1) we get an exact sequence

$$
\begin{aligned}
0 \rightarrow \operatorname{Hom}_{\mathcal{O}_{X}}\left(\mathcal{C}_{n}, M\right) \rightarrow & \mathcal{D}^{n}(M) \stackrel{W^{n}}{\longrightarrow} \operatorname{Hom}_{\mathcal{O}_{X}}\left(\mathcal{V}_{X}, M\right) \\
& \rightarrow \operatorname{Ext}_{\mathcal{O}_{X}}^{1}\left(\mathcal{C}_{n}, M\right) \rightarrow \operatorname{Ext}_{\mathcal{O}_{X}}^{1}\left(\mathcal{P}_{X / k}^{n}(M), M\right) \rightarrow .
\end{aligned}
$$

We can as above identify $\operatorname{Hom}_{\mathcal{O}_{X}}\left(\mathcal{C}_{n}, M\right)$ with the annihilator $\operatorname{Ann}^{n}(V)=\{P \in$ $\left.\mathcal{D}^{n}(M) \mid P \cdot V=0\right\}$, and we will see in Proposition 3.2.2 (4) below that, conversely, if $M$ is a simple $\mathcal{D}$-module, then $V$ can be recovered from Ann $V$.

Clearly, the Taylor map $d_{V, x}^{n}$ is surjective if and only if the evaluation $W_{x}^{n}$ is injective, hence the function $n_{i n j}(x)$ may be defined directly using the map $W^{n}$. In fact, on a regular variety Weierstrass points may equally well be studied using differential operators, as demonstrated in the following result.

Theorem 3.2.1 $\left(X / k\right.$ is a regular variety). Let $M$ be a locally free $\mathcal{O}_{X}$-module, $\mathcal{D}_{X}(M)$ its ring of differential operators and $V$ a separated $k$-subspace of $M_{x}$. Consider the mapping of stalks

$$
W_{x}^{n}: \mathcal{D}^{n}(M)_{x} \rightarrow \operatorname{Hom}_{\mathcal{O}_{x}}\left(\mathcal{V}_{x}, M_{x}\right)=\operatorname{Hom}_{k}\left(V, M_{x}\right) .
$$


Then

$$
n_{\text {inj }}(x)=\min \left\{n \mid W_{x}^{n} \text { is surjective }\right\} .
$$

Proof. Put $s(x)=\min \left\{n \mid W_{x}^{n}\right.$ is surjective $\}$.

$s(x) \leq n_{i n j}(x)$ : Let $n \geq n_{i n j}(x)$. Since $x \mapsto n_{i n j}(x)$ is upper semi-continuous (Proposition 2.2.10), $n \geq N_{i n j}$ so $\mathrm{d}_{V, x}^{n}$ is injective. We may therefore use the sequence (3.3), so $W_{x}^{n}$ is surjective if $\operatorname{Ext}_{\mathcal{O}_{x}}^{1}\left(\mathcal{C}_{x}^{n}, M_{x}\right)=0$. Since $X$ is regular and $M$ is locally free, it follows that $\mathcal{P}_{X / k, x}^{n}(M)$ is free. By Proposition 2.2.1, $n \geq n_{i n j}(x)$ implies that $\mathrm{d}_{V, x}^{n}$ is split injective, so $\mathcal{C}_{x}^{n}$ is free; hence $\operatorname{Ext}_{\mathcal{O}_{x}}^{1}\left(\mathcal{C}_{x}^{n}, M_{x}\right)=0$.

$s(x) \geq n_{i n j}(x)$ : Let $n \geq s(x)$. We first prove that $s(x) \geq N_{i n j}$. Let $N^{*}=$ $\operatorname{Hom}_{\mathcal{O}_{x}}\left(N_{x}, \mathcal{O}_{x}\right)$ when $N_{x}$ is an $\mathcal{O}_{x}$-module. Consider the Taylor sequence (2.1) localised at the point $x$, and let $\mathcal{I}_{x}$ be the image of $\mathrm{d}_{V, x}^{n}$, so we have a surjection $\phi: \mathcal{V}_{x} \rightarrow \mathcal{I}_{x}$. Now since $\mathcal{V}_{x}$ is torsion free, it follows that $\mathcal{V}_{x}=\mathcal{I}_{x}$ if also the dual map $\phi^{*}: \mathcal{I}_{x}^{*} \rightarrow \mathcal{V}_{x}^{*}$ is surjective, which implies that $n \geq N_{i n j}$ since $X$ is irreducible. To see that $\phi^{*}$ is surjective, we first note that by assumption the composed map $\mathcal{D}_{X / k, x}^{n}(M) \rightarrow \operatorname{Hom}_{\mathcal{O}_{x}}\left(\mathcal{I}_{x}, M_{x}\right) \rightarrow \operatorname{Hom}_{\mathcal{O}_{x}}\left(\mathcal{V}_{x}, M_{x}\right)$ is surjective. Hence, since

$M_{x}$ is free, the composed map $\mathcal{P}_{X / k}^{n}(M)^{*} \rightarrow \mathcal{I}_{x}^{*} \stackrel{\phi^{*}}{\longrightarrow} \mathcal{V}_{x}^{*}$ is surjective, hence $\phi^{*}$ is surjective.

If now $n \geq s(x) \geq N_{i n j}$, then, from (3.3), we have a split short exact sequence

$$
0 \rightarrow \operatorname{Hom}_{\mathcal{O}_{x}}\left(\mathcal{C}_{x}^{n}, M_{x}\right) \rightarrow \mathcal{D}^{n}(M)_{x} \stackrel{W_{x}^{n}}{\longrightarrow} \operatorname{Hom}_{\mathcal{O}_{x}}\left(\mathcal{V}_{x}, M_{x}\right) \rightarrow 0
$$

and since $M_{x}$ is free, we have a split exact sequence

$$
0 \rightarrow \operatorname{Hom}_{\mathcal{O}_{x}}\left(\mathcal{C}_{x}^{n}, \mathcal{O}_{x}\right) \rightarrow \operatorname{Hom}_{\mathcal{O}_{x}}\left(\mathcal{P}_{X / k, x}^{n}(M), \mathcal{O}_{x}\right) \rightarrow H_{o m} m_{\mathcal{O}_{x}}\left(\mathcal{V}_{x}, \mathcal{O}_{x}\right) \rightarrow 0
$$

That $X / k$ is regular implies that the $\mathcal{O}_{x}$-module $\mathcal{P}_{X / k, x}^{n}(M)$ is free and hence reflexive, so upon applying $\operatorname{Hom}_{\mathcal{O}_{x}}\left(\cdot, \mathcal{O}_{x}\right)$ to the previous exact sequence we get that the sequence

$$
0 \rightarrow \mathcal{V}_{x} \stackrel{\mathrm{d}_{V, x}^{n}}{\longrightarrow} \mathcal{P}_{X / k, x}^{n}(M) \rightarrow \mathcal{C}_{x}^{n} \rightarrow 0
$$

is split exact. Then Proposition 2.2.1 implies that $n \geq n_{\text {inj }}(x)$, whence $s(x) \geq$ $n_{i n j}(x)$. This completes the proof.

Proposition 3.2.2. Let $X / k$ be a regular and geometrically integral variety and $x$ a point in $X$.

(1) The map $W_{x}: \mathcal{D}_{X / k, x}(M) \rightarrow \operatorname{Hom}_{k}\left(V, M_{x}\right)$ is surjective for each finitedimensional $k$-subspace $V \subset M_{x}$.

(2) $M_{x}$ is an absolutely simple $\mathcal{D}_{X / k, x}\left(M_{x}\right)$-module.

(3) $\operatorname{End}_{\mathcal{D}_{X / k, x}(M)} M_{x}=k$.

(4) Let $L$ be a left ideal of $\mathcal{D}_{X / k}(M)$ such that $\mathcal{D}_{X / k}(M) / L$ is abstractedly isomorphic to $\mathrm{Hom}_{k}(V, M)$ for some finite-dimensional $k$-subspace $V$ (as $\mathcal{D}_{X}(M)$-modules, where $H_{o m}(V, M)$ has the structure given by $\operatorname{Pf}(v)=$ $P(f(v))$, for $\left.P \in \mathcal{D}_{X / k}(M), \quad f \in \operatorname{Hom}_{k}(V, M)\right)$. Then there is a finitedimensional vector space $V_{1} \subset M$, of the same dimension as $V$, such that $L=\operatorname{Ann} V_{1}$. Define the map

$$
S: \operatorname{Hom}_{\mathcal{D}_{X / k}(M)}\left(\mathcal{D}_{X / k}(M) / L, M\right) \rightarrow M, f \mapsto f(1 \bmod L) .
$$

Then $S$ is injective and $V_{1}$ may be selected as the image of $S$. 
Proof. That $(1)-(3)$ are equivalent is proven in [6, 2.6.5]. (1) follows from Theorem 3.2 .1 .

(4) Let $v_{i}^{*}, i=1, \ldots, n$, be a basis of the dual space $V^{*}$. Let $\phi: \mathcal{D}_{X}(M) / L \cong$ $\operatorname{Hom}_{k}(V, M)$ be an isomorphism and $p_{i}: \operatorname{Hom}_{k}(V, M) \cong V^{*} \otimes_{k} M \mapsto M$ be the projection $v_{j}^{*} \otimes m \mapsto \delta_{i j} m ; \mathcal{D}_{X}(M)$ acts trivially on the first factor in $V^{*} \otimes_{k} M$. Then $p_{i} \circ \phi: \mathcal{D}_{X}(M) / L \rightarrow M$ is a surjection. Put $m_{i}=p_{i} \circ \phi(1 \bmod L)$ and $V_{1}=k m_{1}+\cdots+k m_{n}$. Then clearly, $L \subset$ Ann $V_{1}, V_{1}$ has the same dimension as $V$ and by (1) $\mathcal{D}_{X}(M) / \operatorname{Ann} V_{1}=\operatorname{Hom}_{k}\left(V_{1}, M\right) \cong \mathcal{D}_{X}(M) / L$. Therefore $L=$ Ann $V_{1}$. Finally,

$$
\begin{aligned}
\operatorname{Hom}_{\mathcal{D}_{X}(M)}\left(\mathcal{D}_{X}(M) / L, M\right) \cong \operatorname{Hom}_{\mathcal{D}_{X}(M)}\left(V_{1}^{*} \otimes_{k} M, M\right) & \\
& \cong \operatorname{Hom}_{\mathcal{D}_{X}(M)}(M, M) \otimes_{k} V_{1} \cong V_{1}
\end{aligned}
$$

where the last isomorphism follows from (3).

Remark 3.2.3. If $X$ is not regular, it is well-known that $\mathcal{O}_{X}$ need not be a simple $\mathcal{D}_{X}=\mathcal{D}_{X}\left(\mathcal{O}_{X}\right)$-module. Notice that the surjectivity in (1) in Proposition 3.2 .2 for large $n$ follows from the density theorem, if $k$ is algebraically closed and $M_{x}$ is simple holonomic over $\mathcal{D}_{X}$. Thus we have a counterpart of the surjectivity (1) for any simple coherent $\mathcal{D}_{X}(M)$-module, not necessarily coherent over $\mathcal{O}_{X}$. The study of such $\mathcal{D}_{X}$-modules forms one of the most interesting parts of $D$-module theory, so it has some appeal extending the study to holonomic $\mathcal{D}_{X}$-modules.

The next theorem follows from Theorem 3.2.1, together with the identification of Ker $W^{n}$ with $\operatorname{Ann}^{n} V$, discussed earlier.

Theorem 3.2.4. Let $X / k$ be a regular variety, $M$ a locally free $\mathcal{O}_{X}$-module, $\mathcal{D}_{X / k}^{n}(M)$ its $\mathcal{O}_{X}$-module of differential operators of order $n$, and $V$ a finite-dimensional separated $k$-subspace of $\Gamma(X, M)$ (Definition 2.2.6). If $n \geq n_{\text {inj }}(V)$, then one has a locally split short exact sequence

$$
0 \rightarrow \operatorname{Ann}^{n} V \rightarrow \mathcal{D}_{X / k}^{n}(M) \rightarrow V^{*} \otimes_{k} M \rightarrow 0 .
$$

3.3. The sheaf $\mathcal{D}^{V}(M)$. We will now study the sheaf of rings $\mathcal{D}^{V}(M)$ of differential operators that preserve a finite-dimensional vector space $V \subset \Gamma(X, M)$. Notice that

$$
\operatorname{Hom}_{\mathcal{O}_{X}}\left(\mathcal{V}_{X}, M\right)=H_{o m}(V, M)=V^{*} \otimes_{k} M
$$

where $V^{*}=\operatorname{Hom}_{k}(V, k)$ and $\operatorname{End}_{k}(V) \subset \operatorname{Hom}_{\mathcal{O}_{X}}(V, M)$.

\section{Definition 3.3.1.}

$$
\mathcal{D}^{V, n}(M)=\left\{P \in \mathcal{D}_{X / k}^{n}(M) \mid P \cdot V \subset V\right\}=\left(W_{n}^{*}\right)^{-1}\left(\operatorname{End}_{k}(V)\right)
$$

and $\mathcal{D}^{V}(M)=\bigcup_{n \geq 0} \mathcal{D}^{V, n}(M)$ (this is a sheaf of $k$-algebras).

Letting $n$ be an integer $\geq n_{i n j}$, one gets the short exact sequence (3.4), which can be pushed out to the short exact sequence

$$
0 \rightarrow \operatorname{Ann}^{n} V \rightarrow \mathcal{D}^{V, n}(M) \stackrel{W^{n}}{\longrightarrow} \operatorname{End}_{k}(V) \rightarrow 0 .
$$

For obvious reasons this sequence is locally split exact, so let us describe explicitly a splitting. Put $r=\operatorname{dim} V$ and let $\left\{\hat{L}^{i}\right\}$ be a basis of $\operatorname{End}_{k}(V)$. Then select $L_{x}^{i} \in$ $\mathcal{D}_{x}^{V, n_{\text {inj }}}(M)$ such that $W^{n}\left(L_{x}^{i}\right)=\hat{L}^{i}$ and define a local splitting $\phi_{n}: \mathcal{D}_{x}^{V, n}(M) \rightarrow$ $\mathrm{Ann}^{n} V, P_{x} \mapsto P_{x}-\sum_{i} \alpha_{i} L_{x}^{i}$ (the sum contains $r^{2}$ terms), where the coefficients $\alpha_{i} \in k$ satisfy the equation $\sum_{i} \alpha_{i} \hat{L}^{i}=W^{n}\left(P_{x}\right)$ in $\operatorname{End}_{k}(V)$. (Notice that the 
same $\alpha_{i}=\alpha_{i}\left(W^{n}\left(P_{x}\right)\right)$ works for all $x$ in affine subsets of $X$; see the proof of Theorem 3.3 .3 below.) We thus need to compute $r^{2}$ differential operators $L_{x}^{i}$ to define a split.

Remark 3.3.2. The splits $\phi_{n}$ do not preserve the order filtrations of Ann $V$ and $\mathcal{D}^{V}(M)$ when $n<n_{i n j}$. But if $n \geq n_{i n j}$, they do preserve the order filtration in the strong sense that $\phi_{n}$ induces an isomorphism $\mathcal{D}^{V, n} / \mathcal{D}^{V, n-1} \cong \mathrm{Ann}^{n} V / \mathrm{Ann}^{n-1} V$; cf. also [21].

We collect in the following theorems our general results on the irreducibility of the action of $\mathcal{D}^{V}$ on $V$. For $M=\mathcal{A}_{\mathbf{R}^{m}}$ the sheaf of real-valued analytic functions it was proven in [loc. cit., Th. 4.8], expressed in our terminology, that the map $W^{n}(\Omega)$ is surjective for an arbitrary open subset $\Omega$ of some $\mathbf{R}^{m}$ when $V$ is a subspace of $\mathcal{A}_{\mathbf{R}^{m}}(\Omega)$ such that $n_{i n j}(V)<\infty$ and when $n \geq n_{i n j}$. The following result allows locally free modules $M$ of rank $\geq 1$ and affine algebraic varieties defined over arbitrary fields of characteristic zero. The proof is at this point almost a formality.

Theorem 3.3.3. Let $X / k$ be a regular affine variety and $M$ a locally free $\mathcal{O}_{X}$ module. Let $V$ be a separated subspace of $\Gamma(X, M)$. Then if $n \geq n_{\text {inj }}$, we have a short exact sequence

$$
0 \rightarrow \Gamma\left(X, \operatorname{Ann}^{n}(M)\right) \rightarrow \Gamma\left(X, \mathcal{D}^{n, V}(M)\right) \stackrel{W^{n}(X)}{\longrightarrow} \operatorname{End}_{k}(V) \rightarrow 0
$$

and hence an exact sequence

$$
0 \rightarrow \Gamma(X, \operatorname{Ann}(M)) \rightarrow \Gamma\left(X, \mathcal{D}^{V}(M)\right) \stackrel{W(X)}{\longrightarrow} \operatorname{End}_{k}(V) \rightarrow 0 .
$$

Proof. By Theorem 3.2.4 the sequence (3.4) is locally split as vector spaces over $k$, hence if $X$ is affine, also globally split by Serre's vanishing cohomology theorem. Therefore the push-out (3.6) is also split exact. We get (3.7), since a split $\operatorname{End}_{k}(V) \rightarrow \mathcal{D}^{n, V}(X)$ also gives a split $\operatorname{End}_{k}(V) \rightarrow \mathcal{D}^{V}(X)$.

This theorem implies that any geometrically integral real affine variety (i.e., there are no rational functions $f$ such that $f^{2}=-1$ ) has the property that each map $V \rightarrow V$ is realised by a global differential operator on $M$. Hodge algebra, the main component in the proof of 21] of the corresponding result for open subsets of some $\mathbf{R}^{n}$, is here replaced by the fact that affine sets have vanishing higher cohomology.

By instead considering real points $X(\mathbf{R})$ on a variety $X / \mathbf{R}$ (or replacing $\mathbf{R}$ by any ordered field), and its sheaf $\mathcal{O}_{X(\mathbf{R})}$ of rational functions we get a stronger result since $X(\mathbf{R})$ often is affine, e.g. when $X / \mathbf{R}$ is quasi-projective [2, Th. 3.4.4]; $\left(X(\mathbf{R}), \mathcal{O}_{X(\mathbf{R})}\right)$ is an example of a real algebraic variety [loc. cit]. A section of $\mathcal{O}_{X(\mathbf{R})}$ is locally a quotient $p / q$ of regular functions where $q$ does not vanish in any real point. In the same way, differential operators $\mathcal{D}_{X(\mathbf{R})}$ have the form $\sum_{\alpha} f_{\alpha} \partial^{\alpha}$ where $f_{\alpha} \in \mathcal{O}_{X(\mathbf{R})}$ and $\partial_{x_{i}}\left(x_{j}\right)=\delta_{i j}$ for some regular parameters $x_{i}$.

Let $M_{\mathbf{R}}$ and $\mathcal{D}_{X(\mathbf{R})}\left(M_{\mathbf{R}}\right)$ be the localisation of $M$ and $\mathcal{D}_{X}(M)$ on $X(\mathbf{R})$.

Theorem 3.3.4. Let $X / \mathbf{R}$ be a regular variety such that its associated real algebraic variety $\left(X(\mathbf{R}), \mathcal{O}_{X(\mathbf{R})}\right)$ is affine. Then we have an exact sequence

$$
0 \rightarrow \Gamma\left(X(\mathbf{R}), \operatorname{Ann}\left(M_{\mathbf{R}}\right)\right) \rightarrow \Gamma\left(X(\mathbf{R}), \mathcal{D}_{X(\mathbf{R})}^{V}\left(M_{\mathbf{R}}\right)\right) \stackrel{W(X(\mathbf{R}))}{\longrightarrow} \operatorname{End}_{k}(V) \rightarrow 0 .
$$


Proof. Let $x \in X(\mathbf{R}) \subset X . V$ is separated at rational points (Corollary 2.2.7); hence there exists an integer $n_{i n j}(x)$ such that $W_{x}^{n}$ is surjective when $n \geq n_{i n j}(x)$ (Theorem 3.2.1). Hence $W_{x}^{n}$ is surjective when $x$ belongs to a neighbourhood of $x$ in $X(\mathbf{R})$. By quasi-compactness there exists an integer $n_{i n j}$ such that $W_{x}^{n}$ is surjective for each $x \in X(\mathbf{R})$ when $n \geq n_{i n j}$. We have $\mathcal{D}(M)_{X, x} \cong \mathcal{D}\left(M_{\mathbf{R}}\right)_{X(\mathbf{R}), x}$ when $x \in X(\mathbf{R})$. Hence the associated map

$$
W_{\mathbf{R}}^{n}: \mathcal{D}_{X(\mathbf{R}) / \mathbf{R}}^{n}\left(M_{\mathbf{R}}\right) \rightarrow \operatorname{Hom}_{\mathbf{R}}\left(V, M_{\mathbf{R}}\right)
$$

is surjective when $n \geq n_{i n j}$. This implies that the global map $W_{\mathbf{R}}^{n}(X(\mathbf{R}))$ is surjective by the real version of [15, Prop. II.5.6] (it follows essentially for the reason that the inverse image functor $i^{*}$ for the map $i: X(\mathbf{R}) \rightarrow \mathbf{X}$ takes injective $\mathcal{O}_{X}$-modules to flasque sheaves).

It is not difficult to extend this line of argument to any real-analytic manifold.

Similarly to [21, Th. 4.14] one can now estimate the order of the generators of the annihilator of a separated subspace $V$.

Corollary 3.3.5. Put

$$
A=\Gamma\left(X, \mathcal{O}_{X}\right), \quad U=\Gamma\left(X, \mathcal{D}_{X}(M)\right) \quad \text { and } \quad U^{1}=\Gamma\left(X, \mathcal{D}_{X}^{1}(M)\right) .
$$

Assume that $U$ is generated, as an A-algebra, by $U^{1}$. Let $n$ be an integer such that the sequences (3.6) and (3.7) are split exact. Then the left ideal $\Gamma(X, A n n V) \subset U$ is generated by the A-module $\Gamma\left(X, \mathrm{Ann}^{n+1} V\right)$.

Of course, the assumption on $U$ is always satisfied locally if $X / k$ is regular.

Proof. Put $J^{i}=\Gamma\left(X, \mathrm{Ann}^{i} V\right)$ and $U^{i}=\Gamma\left(X, \mathcal{D}_{X / k}^{i}(M)\right)$. If $m=n+1$, clearly

$$
J^{m} \subset U J^{n+1} \text {. }
$$

This gives us the basis for proving by induction that the inclusion is true for all $m \geq n+1$. Assume then $m>n+1$, and that the inclusion is true for $m-1$. Let $\phi_{m}: U^{m} \rightarrow J^{m}, m=n, n+1, \ldots$, be the splits described after the sequence (3.5). Since $U^{1}$ generates $U$ it follows that if $P \in J^{m} \subset U^{m}, m>n$, then

$$
P=\sum P_{i}^{(1)} P_{i}^{(2)}
$$

where $P_{i}^{(1)} \in U^{m_{1}}$ with $m_{1}<m$ and $P_{i}^{(2)} \in U^{n+1}$. By Remark 3.3.2, $P_{i}^{(2)}-$ $\phi_{n+1}\left(P_{i}^{(2)}\right)=P_{i}^{(2)} \bmod U^{n}$, hence $P_{i}^{(1)} P_{i}^{(2)}-P_{i}^{(1)} \phi\left(P_{i}^{(2)}\right)$ is a differential operator of order $\leq m-1$, i.e.,

$$
P-\sum_{i} P_{i}^{(1)} \phi_{n+1}\left(P_{i}^{(2)}\right) \in J^{m-1},
$$

so $P \in J^{m-1}+U J^{n+1}$, and by induction

$$
J^{m-1} \subset U J^{n+1},
$$

hence $P \in U J^{n+1}$.

In general, in a global situation when Theorem 3.3 .3 is not applicable, it is hard to decide when $V$ is simple as a module over the global differential operators $\Gamma\left(X, \mathcal{D}^{V}(M)\right)$. Still there are interesting cases when one can prove simplicity. It is, for example, true in certain cases both for toric varieties and homogeneous spaces, as will be discussed later. In the case where the ground-field is algebraically closed, this is clearly equivalent, by the density theorem, to $W(X)$ being surjective. Hence 
we can describe the differential operators on $X$ that preserve $V$, in an obvious but important way:

Theorem 3.3.6. Let $M$ be a quasi-coherent $\mathcal{O}_{X}$-module and let $V$ be a subspace of $\Gamma(X, M)$ that is simple as a $\Gamma\left(X, \mathcal{D}_{X}^{V}(M)\right)$-module. Then

$$
\mathcal{D}_{X}^{V}(M)=\Gamma\left(X, \mathcal{D}_{X}^{V}(M)\right)+\operatorname{Ann}_{\mathcal{D}_{\mathrm{X}}(\mathrm{M})}(\mathrm{V})
$$

(equality of sheaves, where $\Gamma\left(X, \mathcal{D}_{X}^{V}(M)\right)$ is regarded as the constant sheaf of global sections of $\left.\mathcal{D}_{X}^{V}(M)\right)$.

\section{Completions}

Finite-dimensional vector spaces of functions $V$ occur often as linear systems associated to invertible sheaves on proper varieties. Furthermore, the differential operators that preserve these spaces, considered as spaces of functions on an open subset, stem from important examples, from differential operators on the proper variety. In this section we will explore conditions on $V$ that ensure that this situation occurs. First we will recall the well-known method of constructing a proper variety from a finite-dimensional subspace of a $k$-algebra. To simplify the use we have given full references and some arguments. The reader already familiar with this can read the definition below, and then skip to Section 4.2.

4.1. Linear systems. To fix our situation and keep track of our maps we define a category $\mathcal{C}$ as follows. An object $A$ in $\mathcal{C}$ consists of the datum $\left(X, \mathcal{O}_{X}, V \stackrel{i}{\rightarrow}\right.$ $\Gamma(X, M), M)$ where $\left(X, \mathcal{O}_{X}\right)$ is a variety over the field $k, V$ is a finite-dimensional vector space over $k$ and $i$ is a $k$-linear injective map to the global sections $\Gamma(X, M)$ of a locally free module $M$. Let $A=\left(X, \mathcal{O}_{X}, V \stackrel{i}{\rightarrow} \Gamma(X, M), M\right)$ and $B=$ $\left(X^{\prime}, \mathcal{O}_{X^{\prime}}, V^{\prime} \stackrel{i^{\prime}}{\rightarrow} \Gamma\left(X, M^{\prime}\right), M^{\prime}\right)$ be objects in $\mathcal{C}$. A morphism $J: A \rightarrow B$ is a morphism of $k$-varieties $j: X \rightarrow X^{\prime}$, an isomorphism of $\mathcal{O}_{X}$-modules $\psi: j^{*}\left(M^{\prime}\right) \rightarrow M$, and an induced surjective map of linear spaces $F=\Gamma(\psi): V^{\prime} \rightarrow V$.

If $j: X \subset X^{\prime}$ is the inclusion of an open subset, then the restriction $B_{\mid X}:=$ $\left(X, \mathcal{O}_{X}, V \rightarrow \Gamma\left(X, j^{*} M\right), j^{*} M\right)$ belongs to $\mathcal{C}$, and there is clearly a morphism $B_{\mid X} \rightarrow B$.

Definition 4.1.1. An object $B=\left(X^{\prime}, \mathcal{O}_{X^{\prime}}, V^{\prime} \stackrel{i^{\prime}}{\rightarrow} \Gamma\left(X, M^{\prime}\right), M^{\prime}\right)$ in $\mathcal{C}$ is a completion of $A=\left(X, \mathcal{O}_{X}, i: V \rightarrow \Gamma(X, M), M\right)$ if there is a morphism $A \rightarrow B$ in $\mathcal{C}$ such that

(1) $j: X \rightarrow X^{\prime}$ is an open immersion into a projective variety $X^{\prime}$;

(2) $i^{\prime}: V^{\prime} \rightarrow \Gamma\left(X^{\prime}, M^{\prime}\right)$ is an injection;

(3) $F: V^{\prime} \rightarrow V$ is an isomorphism.

If in addition $i^{\prime}$ is an isomorphism, then the completion is full.

Thus a completion is a simultaneous extension of $X$ to a projective variety and a locally free extension $\bar{M}$ of $M$, with the condition that $V \subset \Gamma\left(X^{\prime}, M^{\prime}\right)$, and it is full if $V \cong \Gamma\left(X^{\prime}, M^{\prime}\right)$. If $j: X \subset X^{\prime}$ is the inclusion of an open subset of a projective variety, we see that $B_{\mid X}$ thus has the completion $B$, and it is a full completion if $B=\left(X^{\prime}, \mathcal{O}_{X^{\prime}}, \Gamma\left(X, M^{\prime}\right) \stackrel{I d}{\longrightarrow} \Gamma\left(X, M^{\prime}\right), M^{\prime}\right)$.

First, we will use the standard algebraic geometric methods to construct a canonical completion $C(A)$, as appropriate in our situation; cf. [15, 13. We will only 
consider objects in $\mathcal{C}$ of the form $A=(X, R, V \subset R, R)$ where $X=\operatorname{Spec} R$ is integral affine of finite type over $k$.

Definition 4.1.2. Let $V \subset R$ be a finite-dimensional vector subspace, and let

$$
\gamma: \mathrm{S}[V][t] \rightarrow R[t]
$$

where $\mathrm{S}[V]$ is the symmetric algebra, be the graded homomorphism between rings graded by degree in $t$. Let $B_{V}$ be the subalgebra of $S[V][t]$ that is generated by $t V$ and denote by $A_{V}$ the image $\operatorname{Im} \gamma\left(B_{V}\right)$. Define

$$
X_{V}:=\operatorname{Proj} A_{V}
$$

and let $\mathcal{L}_{V}=\mathcal{O}_{X_{V}}(1)$ be the associated invertible.

Note that $X_{V}$ is a closed projective subvariety of $\operatorname{Proj}(\mathrm{S}[t V])=\mathbf{P}^{(n-1)}$, if $\operatorname{dim}_{k} V=n$ and that $\mathcal{L}_{V}$ is the pullback of $\mathcal{O}_{\mathbf{P}^{(n-1)}}(1)$, and hence very ample.

There is by definition an inclusion $i: A_{V} \rightarrow R[t]$, and hence a rational map $i: X=\operatorname{Proj} R[t] \rightarrow X_{V}$, defined on $X-V_{+}\left(R[t] i\left(\left(A_{V}\right)_{+}\right)\right)$(cf.[13, 3.1.7, 2.8.1]; $V_{+}()$denotes the closed subvariety defined by a graded ideal). Now

$$
R[t] i\left(\left(A_{V}\right)_{+}\right)=R \oplus V R t \oplus V R t^{2} \oplus \ldots
$$

( $V R$ is the ideal generated by $V)$ and by [13, 2.3.13], $V_{+}\left(R[t] i\left(\left(A_{V}\right)_{+}\right)\right)$is empty if and only if every element of $R[t]_{+}$has a power that is contained in $R[t] i\left(\left(A_{V}\right)_{+}\right)$. Applying this to $t \in R[t]_{+}$we find that $V R=R$. Hence $i$ is defined on the whole of $X$ if and only if the following holds:

Condition $I$. The ideal $V R=R$. (This is trivially true in the case that $V$ contains a unit.)

The map $i$ is furthermore an open immersion if, in addition, the following condition holds (it is not the most general condition possible, but sufficient for our purposes):

Condition II.

$$
\left(\left(A_{V}\right)_{t v}\right)_{0} \cong \gamma\left(S\left[V v^{-1}\right]\right)=R_{v},
$$

for a set of $v=v_{i}, i=1, \ldots, r$, such that $\left(v_{1}, \ldots, v_{r}\right)=R$. If $v \in V$ is a unit in $R$, and $\gamma$ is surjective, then actually $X=\operatorname{Spec} R \cong D_{+}(t v)$, since $\left(\left(A_{V}\right)_{t v}\right)_{0}=R$, and Condition II is trivially satisfied.

That Condition II implies that $i$ is an open immersion is easily checked, since Proj $A_{V}$ is constructed by glueing together the affine spaces

$$
D_{+}(t v):=\operatorname{Spec}\left(\left(A_{V}\right)_{t v}\right)_{0} \cong \gamma\left(S\left[V v^{-1}\right]\right),
$$

for sections $t v \in t V \subset A_{V}$, and hence the isomorphism in the condition only says that $i$ induces an isomorphism $D_{+}(t v) \cong D(v) \subset \operatorname{Spec} R$, and that $D\left(v_{i}\right), i=$ $1, \ldots, r$, cover $\operatorname{Spec} R$.

Assuming that Conditions I and II hold, put

$$
C(A):=\left(X_{V}, \mathcal{O}_{X_{V}}, V \stackrel{i}{\rightarrow} \Gamma\left(X_{V}, \mathcal{L}_{V}\right), \mathcal{L}_{V}\right) .
$$

There is a morphism $A \rightarrow C(A)$ given by $i: X \rightarrow X_{V}, \psi: i^{*}\left(\mathcal{O}_{X_{V}}(1)\right) \cong$ $\mathcal{O}_{\text {Proj } R[t]}(1) \cong\left(\mathcal{O}_{X}, F: V \stackrel{I d}{\longrightarrow} V\right.$.

Proposition 4.1.3. Let $X=\operatorname{Spec} R$ be integral and assume that $A:=\left(X, \mathcal{O}_{X}\right.$, $\left.V \stackrel{i}{\rightarrow} \Gamma\left(X, \mathcal{O}_{X}\right), \mathcal{O}_{X}\right) \in \mathcal{C}$ satisfies Conditions I and II. 
(1) The canonical completion $C(A)$ is full if and only if the restriction map

$$
V=\Gamma\left(\mathbf{P}^{(n-1)}, \mathcal{O}_{\mathbf{P}^{(n-1)}}(1)\right) \rightarrow \Gamma\left(X_{V}, \mathcal{L}_{V}\right)
$$

is surjective. This is true if, e.g., $A_{V}$ is normal.

(2) There is a finite-dimensional vector space $V \subset V^{*} \subset R$ such that $A^{*}:=$ $\left(X, \mathcal{O}_{X}, V^{*} \stackrel{i}{\rightarrow} \Gamma\left(X, \mathcal{O}_{X}\right), \mathcal{O}_{X}\right)$ has a full completion, in fact, $\left(X_{V}, \mathcal{O}_{X}\right.$, $\left.\Gamma\left(X_{V}, \mathcal{L}_{V}\right) \stackrel{I d}{\longrightarrow} \Gamma\left(X_{V}, \mathcal{L}_{V}\right), \mathcal{L}_{V}\right)$ is such a full completion.

(3) Any full completion of $A, B=\left(\bar{X}, \mathcal{O}_{\bar{X}}, \Gamma(\bar{X}, \mathcal{L}) \stackrel{I d}{\longrightarrow} \Gamma(\bar{X}, \mathcal{L}), \mathcal{L}\right)$, where $\mathcal{L}$ is very ample, satisfies $\bar{X} \cong X_{V}, \mathcal{L} \cong \mathcal{L}_{V}$.

Proof. (1) The restriction map may be identified with $\gamma$, which makes the first assertion obvious. Since $A_{V}$ is the homogeneous coordinate ring for the embedding of $X_{V}$ in $\mathbf{P}^{(n-1)}$, the assertion on normality is contained in [15, Exc. II.5.14 (d)].

(2) Put $V^{*}:=\Gamma\left(X_{V}, \mathcal{L}_{V}\right)_{\mid X}$. Observe first that the restriction map

$$
\mathrm{S}^{m}(V)=\Gamma\left(\mathbf{P}^{(n-1)}, \mathcal{O}(m)\right) \rightarrow \Gamma\left(X_{V}, \mathcal{L}_{V}^{m}\right)
$$

is surjective for $m$ large enough, since $\mathcal{O}(1)$ is ample. Note further that $R[t] \cong$ $\bigoplus_{m \geq 0}^{\infty} \Gamma\left(X, \mathcal{L}^{m}\right) t^{m}$, using the isomorphism $\psi: j^{*}\left(\mathcal{L}_{V}\right) \rightarrow \mathcal{O}_{X}$, and that the restriction map hence gives an injection $\Gamma^{*}:=\bigoplus_{m \geq 0}^{\infty} \Gamma\left(X_{V}, j^{*}\left(\mathcal{L}_{V}^{m}\right)\right) \subset R[t]$, compatible with multiplication. The composition $\bigoplus_{m \geq 0} \mathrm{~S}^{m}(V) \rightarrow R[t]$ has the image $A_{V}$, which thus is the homogeneous coordinate ring of $X_{V}$, and coincides with the graded ring $\Gamma^{*}$ in high enough degrees. Furthermore, $A_{V^{*}}$ is the image of the algebra $k\left[V^{*}\right]$ generated by $V^{*}$ in $R[t]$, and it clearly contains $A_{V}$ and hence $\left(A_{V^{*}}\right)_{m}=\left(A_{V}\right)_{m}$, for $m$ large enough and consequently $\operatorname{Proj}\left(A_{V^{*}}\right) \cong \operatorname{Proj}\left(A_{V}\right)$, and $\mathcal{L}_{V} \cong \mathcal{L}_{V^{*}}$.

(3) This follows in a similar way. There is an injection

$$
\Gamma^{*}(\mathcal{L}):=\bigoplus_{m \geq 0} \Gamma\left(\bar{X}, \mathcal{L}^{m}\right) t^{m} \subset R[t] .
$$

Since $V=\Gamma(\bar{X}, \mathcal{L})$ and $\mathcal{L}$ is very ample, the graded algebra $k[V]$ generated by $V$ in $\Gamma_{*}$ coincides with $A_{V}$ in $R[t]$, and hence $X_{V} \cong \operatorname{Proj}\left(A_{V^{*}}\right) \cong \operatorname{Proj}\left(\Gamma_{*}\right) \cong \bar{X}$ (the last equality by very ampleness of $\mathcal{L})$. Similarly, we recover $\mathcal{L}$.

4.2. Extending differential operators. We will give a condition on a finitedimensional vector space $V \subset R$, that ensures that a differential operator preserving $V$ actually stems from a differential operator preserving an invertible module on a proper variety.

Theorem 4.2.1. Let $X$ be a variety, and $M$ a locally free $\mathcal{O}_{X}$-module. Let $J$ : $A \rightarrow B$ be a full completion, where

$$
A=\left(X, \mathcal{O}_{X}, i: V \rightarrow \Gamma(X, M), M\right) \text { and } B=\left(\bar{X}, \mathcal{O}_{X}, \bar{i}: \bar{V} \cong \Gamma(\bar{X}, \bar{M}), \bar{M}\right) .
$$

Then the restriction map $\Gamma\left(\bar{X}, \mathcal{D}_{\bar{X}}^{n}(\bar{M})\right) \rightarrow \Gamma\left(X, \mathcal{D}^{n}(M)\right)$ takes its values in

$$
\Gamma\left(X, \mathcal{D}_{X}^{n, V}(M)\right)
$$

i.e., we have an injective map

$$
r_{\bar{X}, X}: \Gamma\left(\bar{X}, \mathcal{D}_{\bar{X}}^{n}(\bar{M}) \rightarrow \Gamma\left(X, \mathcal{D}_{X}^{n, V}(M)\right) .\right.
$$

If $n \leq n_{s}^{1}$ (Definition 2.3.1), i.e., $\operatorname{codim}_{\bar{X}} \operatorname{supp}_{\bar{X}}^{n} \geq 2$, then $r_{\bar{X}, X}$ is an isomorphism. If $\bar{M}$ is very ample, then, in particular, it is always an isomorphism for $n=1$. 
Proof. We have $\Gamma\left(\bar{X}, \mathcal{D}_{\bar{X}}^{n}(\bar{M})\right)=\Gamma\left(\bar{X}, \mathcal{D}_{\bar{X}}^{n, \bar{V}}\right)$. Since $\bar{M}$ is torsion free, it follows that its ring of differential operators $\mathcal{D}_{\bar{X}}(\bar{M})$ also is torsion free, so the restriction map $\Gamma\left(\bar{X}, \mathcal{D}_{\bar{X}}^{n}(\bar{M})\right) \rightarrow \Gamma\left(X, \mathcal{D}_{X}^{n}(M)\right)$ is injective, and by the previous sentence its values preserve $\bar{V} \cong V$. This proves the first assertion.

Assume $n \leq n_{\text {surj }}^{1}$. An element $P$ in $\mathcal{D}^{n, V}(X)$ gives an element $\tilde{P}$ in

$$
\operatorname{Hom}_{\mathcal{O}_{\bar{X}}}\left(\mathcal{V}_{\bar{X}}, \bar{M}\right)=\operatorname{Hom}_{k}(V, \bar{M}) .
$$

The fact that $\tilde{P}$ comes from $P$ implies that generically the kernel $\mathcal{K}^{n}$ of $\mathrm{d}_{V}^{n}$ : $\mathcal{V}_{\bar{X}} \rightarrow \mathcal{P}_{\bar{X} / k}^{n}(\bar{M})$ belongs to the kernel of $\tilde{P}$, but since $\mathcal{V}_{\bar{X}}$ and $\bar{M}$ are locally free, this gives $\mathcal{K}^{n} \subset \operatorname{Ker} \tilde{P}$. Hence $\tilde{P}$ gives an element $\bar{P}$ in $\operatorname{Hom}_{\mathcal{O}_{\bar{X}}}\left(\operatorname{Im~d}_{V}^{n}, \bar{M}\right)$. Since $\mathrm{d}_{V}^{n}$ is surjective at points of height $1, \bar{P}$ defines a map from $\mathcal{P}_{\bar{X} / k}^{n}(\bar{M})$ to $\bar{M}$ outside the codimension $\geq 2$ subset $\operatorname{supp} \mathcal{C}_{\bar{X}}^{n}$ of $\bar{X}$. Now $\bar{M}$ is locally free, so $\bar{M}_{x}$ has depth $\geq 2$ when $x \in \operatorname{supp} \mathcal{C}_{\bar{X}}^{n}$, hence $\bar{P}^{n}$ actually gives an element in $\operatorname{Hom}_{\mathcal{O}_{\bar{X}}}\left(\mathcal{P}_{\bar{X} / k}^{n}(\bar{M}), \bar{M}\right)=\mathcal{D}_{\bar{X}}^{n}(\bar{M})(\bar{X})$.

The last assertion follows from the result earlier (Lemma 2.3.2) that very ampleness of a line-bundle implies that $n_{\text {surj }}^{1} \geq 1$ for the global sections.

Corollary 4.2.2. Keep the assumptions in Theorem 4.2.1 and assume also that $\operatorname{dim}_{k} \mathcal{O}_{X}(X)=\infty$ (e.g. $X$ is affine). If $n \leq n_{\text {surj }}^{1}$, then $\mathrm{Ann}^{n} V=0$.

Proof. By Theorem 4.2.1, $\Gamma\left(X, \mathcal{D}_{X}^{n, V}(M)\right)$ is finite-dimensional over $k$, hence $\operatorname{dim}_{k} \operatorname{Ann}^{n} V<\infty$. However, $\operatorname{Ann}^{n} V$ is a torsion free $\mathcal{O}_{X}(X)$-module, implying that $\mathrm{Ann}^{n} V=0$.

\section{Hidden Lie AlGeBras}

In this section we will consider the situation when $V \subset A=\mathcal{O}_{X_{0}}$ is invariant under a reductive Lie algebra of differential operators in $\mathcal{D}_{X_{0}}^{1}$. General references for representation theory and homogeneous spaces are $[19,22,16$. We will assume that $k$ is algebraically closed.

5.1. An enlightening example. Consider $X:=\mathbf{A}^{n}$ and $V_{m}=\left\langle\prod_{i=1}^{n} x_{i}^{k_{i}}\right| 0 \leq k_{i}$ and $\left.\sum k_{i} \leq m\right\rangle$. We get that Conditions I and II of the preceding section are fulfilled and clearly $\bar{X}_{V}=\mathbf{P}^{n}$ and $\mathcal{L}_{V_{m}}=\mathcal{O}(m)$. Thus

$$
C(A)=\left(\mathbf{P}^{n}, \mathcal{O}_{\mathbf{P}^{n}}, V_{m} \cong \Gamma\left(\mathbf{P}^{n}, \mathcal{O}(m)\right), \mathcal{O}(m)\right)
$$

is a full completion of

$$
\left(\mathbf{A}^{n}, \mathcal{O}_{\mathbf{A}^{n}}, V \rightarrow \Gamma\left(\mathbf{A}^{n}, \mathcal{O}_{\mathbf{A}^{n}}\right), \mathcal{O}_{\mathbf{A}^{n}}\right) .
$$

Proposition 5.1.1. (1) The Taylor map is an isomorphism

$$
\mathrm{d}_{V}^{m}: \mathcal{O}_{\mathbf{P}^{n}} \otimes_{k} V_{m} \cong \mathcal{P}_{\mathbf{P}^{n} / k}^{m}(\mathcal{O}(m)) .
$$

(2) $n_{\text {inj }}=n_{\text {surj }}=n_{\text {surj }}^{1}=m$.

Proof. Let $\mathbf{A}^{n}$ with coordinates $x_{1}, \ldots, x_{n}$ be one of the open cells of $\mathbf{P}^{n}$, defined by the section $t$ of $\mathcal{O}(1)$. By (2.2) the fibre of the Taylor map in $x \in \mathbf{A}^{n}$, with maximal ideal $\mathfrak{m}_{x}$, is isomorphic to

$$
V_{\mathfrak{m}_{x}} \rightarrow \mathcal{O}_{\mathbf{A}^{n}} \mathcal{O}_{\mathbf{A}^{n}} / \mathfrak{m}_{x}^{m+1} .
$$


The first map here is just $v \mapsto v / t^{m}$, so the image is just the span of all polynomials of degree less than $m$, and the map is clearly a isomorphism. Hence the Taylor map is an isomorphism, and this implies (2).

(This is also the last step in the proof of Proposition 2.3.4)

Hence the behaviour in each fibre is the same. This can be seen also as a consequence of the fact that $X=\mathbf{P}^{n}$ is a homogeneous space for the group $\operatorname{SL}(n, k)$, added to the fact that the Taylor map for homogeneous spaces and equivariant sheaves is equivariant, and hence surjective or injective in a fibre if and only if it has this property in every fibre. We will return to this in the next section.

Since $\mathbf{P}^{n}$ is a homogeneous space for the group $\operatorname{SL}(n, k)$, there is a map $\beta$ : $U\left(\mathfrak{s l}_{n}\right) \rightarrow \Gamma(X, \mathcal{D}(m))($ see $[22])$.

Corollary 5.1.2 ([30]). (1) Any differential operator that has order less than $m$ and preserves the vector space $V_{m}$ of polynomials of degree less than $m$, is a polynomial in the differential operators $\partial_{x_{l}}, x_{k} \partial_{x_{l}}, k, l=1, \ldots, n$, together with $-\sum_{i=1}^{n} x_{i} x_{k} \partial_{x_{i}}+m x_{k}, k=1, \ldots, n$.

(2) $\mathcal{D}^{V, 1} \cong \mathfrak{s l}_{n} \subset \operatorname{End}_{k}(V)$.

(3) $\beta\left(U\left(\mathfrak{s l}_{n}\right)\right)+\operatorname{Ann} V=\mathcal{D}^{V}$.

Proof. Taking the global sections of $\mathcal{D}_{X}(m)$-modules $V=\Gamma\left(X, \mathcal{O}_{X}(m)\right)$ is a traditional way to construct a finite-dimensional simple $U\left(\mathfrak{s l}_{n}\right)$-module. Hence $V$ is also simple as a $\Gamma(X, \mathcal{D}(m))$-module, so $W$ will be surjective, and Theorem 3.3.6 applies. This gives (3). Furthermore, $n_{\text {surj }}^{1}=m$, so Corollary 4.2.2 is applicable and gives that $\operatorname{Ann}^{n} V=0$ if $n \leq m$, in particular, for $n=1$; this implies (2) (In fact it is easy to see that $\operatorname{Ann}_{\mathcal{D}_{\mathrm{X}}}(\mathrm{V})$ is the left ideal generated by the derivations $\partial^{\alpha}=\prod \partial_{x_{i}}^{\alpha_{i}}$, where $\alpha=\left(\alpha_{1}, \ldots, \alpha\right)_{n}$, and $\sum_{1}^{n} \alpha_{i} \geq n+1$.) Finally, (1) follows from the explicit description of $\beta$; see $25,3,22$.

As a further corollary we can prove Theorem 3.3 .3 in the case when $X=\mathbf{A}^{n}$ with a simple geometric argument. First a lemma:

Lemma 5.1.3. If $V$ is a subspace of $\bar{V}$ such that $\bar{V}$ is simple over $\mathcal{D}^{\bar{V}}$, then $V$ is simple over $\mathcal{D}^{\bar{V}, V}=\mathcal{D}^{V} \cap \mathcal{D}^{\bar{V}}$, the subsheaf of $\mathcal{D}_{X}^{\bar{V}}(M)$ consisting of sections that preserve $V$. Hence it is also simple over $\mathcal{D}^{V}$.

The idea of the easy proof is to choose a vector space complement $\bar{V}=K \oplus V$, which induces an embedding $\operatorname{End}(V) \subset \operatorname{End}(\bar{V})$.

Corollary 5.1.4. Suppose that $V \subset \mathcal{O}_{\mathbf{A}^{n}}\left(\mathbf{A}^{n}\right)=k\left[x_{1}, \ldots, k_{n}\right]$ is a nonzero finitedimensional $k$-subspace.

(1) $V \subset \mathcal{O}_{\mathbf{A}^{n}}\left(\mathbf{A}^{n}\right)$ is simple as $\mathcal{D}^{V}$-module.

(2) There is a completion $\left(\mathbf{P}^{n}, \mathcal{O}_{\mathbf{P}^{n}}, V_{m} \cong \Gamma\left(\mathbf{P}^{n}, \mathcal{O}(m)\right), \mathcal{O}_{\mathbf{P}^{n}}(m)\right)$ for some positive integer $m$, of $\left(\mathbf{A}^{n}, \mathcal{O}_{\mathbf{A}^{n}}, V \subset \mathcal{O}_{\mathbf{A}^{n}}\left(\mathbf{A}^{n}\right), \mathcal{O}_{\mathbf{A}^{n}}\right)$. Furthermore,

$$
\Gamma\left(\mathbf{P}^{n}, \mathcal{D}_{\mathbf{P}^{n} / k}(\mathcal{O}(m))\right)+\operatorname{Ann} V=\mathcal{D}^{V} .
$$

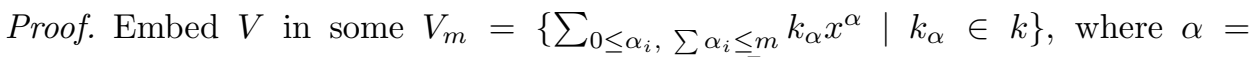
$\left(\alpha_{1}, \ldots, \alpha_{n}\right), x^{\alpha}=x_{1}^{\alpha_{1}} \cdots x_{n}^{\alpha_{n}}$. Then as above $\bar{X}_{V}=\mathbf{P}^{n}$ and $\mathcal{L}_{V}=\mathcal{O}(m)$. The enveloping algebra $\mathrm{U}(\mathfrak{s l}(n, k))$ gives naturally global differential operators on $\mathcal{O}(m)$ that make $V_{m}$ a simple module. Hence $V_{m}$ is simple also as a $\mathcal{D}^{V_{m}}$-module and Lemma 5.1.3, together with Theorem 3.3.6, gives the result. 
5.2. Representations of Lie-algebras. From now on assume that $k$ is algebraically closed. We will now show how these results extend to other homogeneous varieties and representations (as also has been done in [30, without using homogeneous varieties).

First consider representations of a reductive connected and simply-connected (not really necessary) group $G$. Each irreducible representation is constructed as $V=\Gamma(G / B, \mathcal{L})$ for some line bundle on the Borel variety $G / B$, where $\mathcal{L}=\mathcal{L}(\lambda)$ is associated to a unique integral and dominant character $\lambda$ of the torus $T \subset B$ (recall that dominant means that $\langle\lambda, \check{\alpha}\rangle \geq 0$ for all roots $\alpha$ belonging to a basis of the root system consisting of positive roots, and if all inequalities are strict $\lambda$ is regular).

The Borel variety $G / B$ contains an open cell $U$ that is a $B$-orbit, which is isomorphic to some $\mathbf{A}^{n}$, and hence there is an inclusion $V \subset \mathcal{O}_{G / B}(U)=k\left[x_{1}, \ldots, x_{n}\right]=$ : $R$, which is completely specified by the condition that it sends a primitive vector to $1 \in R$. Actually $G / B$ is covered by affine cells $g U$, all isomorphic to $\mathbf{A}^{n}$, so there are many possible embeddings of $V$ in $R$. If $\lambda$ is integral, dominant, and regular, the line bundle is very ample, so the procedure is invertible; we have $X_{V} \cong G / B$ and $\mathcal{L}_{V} \cong \mathcal{L}$ and hence also $\Gamma\left(X_{V}, \mathcal{L}_{V}\right)=V$.

If $\lambda$ is integral dominant, but not regular, there are simple roots $\alpha$ such that $\left\langle\lambda, \check{\alpha}_{i}\right\rangle=0, i \in I$, and they define a parabolic group $P_{I}$. Furthermore, if $\pi: G / B \rightarrow$ $G / P$ is the canonical projection, then $V=\Gamma\left(G / B, \pi^{*}(\mathcal{L})\right) \stackrel{\sim}{\longrightarrow} V=\Gamma(G / P, \mathcal{L})$, for a certain line-bundle $\mathcal{L}$. In this case $X_{V}=G / P$, even if we start with $V \subset R$.

Since $\mathfrak{g}$ will act as first order differential operators (twisted derivations) on $R=$ $\mathcal{O}_{G / B}(U)$, and $V$ is irreducible as a $\mathfrak{g}$-module, the situation of Theorem 3.3.6 holds. It is also well known what the ring of global differential operators of $\mathcal{L}(\lambda)$ is; see [1]. Hence the following is immediate.

Proposition 5.2.1. Suppose that $V \subset R$ is the inclusion of $V=\Gamma(G / B, \mathcal{L}(\lambda))$ that is specified by sending a $B$-primitive vector to 1 in $R$. There is a canonical surjection $r: \mathrm{U}(\mathfrak{g}) \rightarrow \Gamma\left(G / B, \mathcal{D}_{\mathcal{L}(\lambda)}\right)$, whose kernel is the ideal generated by a maximal ideal of the center $Z$ of $\mathrm{U}(\mathfrak{g})$. Hence

$$
\mathcal{D}_{R / k}^{V}=r(\mathrm{U}(\mathfrak{g}))+\operatorname{Ann} V,
$$

and if $n \leq n_{\text {surj }}^{1}$,

$$
\mathcal{D}_{R / k}^{n, V}=r\left(\mathrm{U}^{n}(\mathfrak{g})\right) .
$$

Moreover, if $X=\operatorname{Spec} R$, then $X_{V}=G / B$ and $\mathcal{L}_{V}=\mathcal{L}(\lambda)$.

This description of $\mathcal{D}_{R / k}^{V}$ is the main result of [30].

$G$-equivariant sheaves. Let $G$ be an arbitrary algebraic group, and $X$ a $G$-variety, with the action given by $\mu: G \times X \rightarrow X$. Recall the following induced descent diagram:

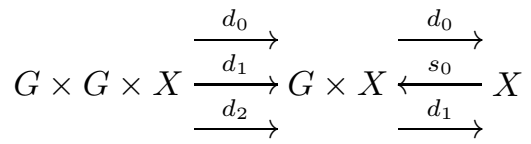

$$
\begin{aligned}
& d_{0}\left(g_{1}, x\right)=g_{1}^{-1} x \quad d_{0}\left(g_{1}, g_{2}, x\right)=\left(g_{2}, g_{1}^{-1} x\right) \\
& d_{1}\left(g_{1}, x\right)=x \quad d_{1}\left(g_{1}, g_{2}, x\right)=\left(g_{1} g_{2}, x\right) \\
& s_{0}(x)=(e, x) \quad d_{2}\left(g_{1}, g_{2}, x\right)=\left(g_{1}, x\right) .
\end{aligned}
$$


Note that all maps here are flat. An $\mathcal{O}_{X}$-module $\mathcal{M}$ is called $G$-equivariant (see 32, 24]), if there is an $\mathcal{O}_{G \times X}$-module isomorphism $\alpha: d_{1}^{*}(\mathcal{M}) \cong d_{0}^{*}(\mathcal{M})$, such that the descent conditions $d_{0}^{*} \alpha \circ d_{2}^{*} \alpha=d_{1}^{*} \alpha$ and $s_{0}^{*} \alpha=i d_{\mathcal{M}}$ are true.

More properly $(M, \alpha)$ is really the object that should be called equivariant, and such objects form, in an obvious way, an abelian category. However, we will abuse notation and call $M$ equivariant. As a module over itself $\mathcal{O}_{X}$ is $G$-equivariant, with structure map $\alpha$ the composition of the canonical isomorphisms $\alpha: d_{0}^{*}\left(\mathcal{O}_{X}\right) \cong$ $\mathcal{O}_{G \times X} \cong d_{1}^{*}\left(\mathcal{O}_{X}\right)$. Below we will need that this map is an a homomorphism of sheaves of rings.

The fact that the principal part bundle of a $G$-equivariant module is $G$-equivariant is folklore; since we have been unable to find a reference, we include for convenience a sketch of the simple proof.

Proposition 5.2.2. Let $X$ be a $G$-variety. If $\mathcal{M}$ is $G$-equivariant, the $\mathcal{O}_{X}$-modules $\mathcal{P}_{X / k}^{n}(\mathcal{M})$ have compatible structures as $G$-equivariant modules. If $V \subset \Gamma(X, \mathcal{M})$ is invariant under the $G$-action induced from the equivariant structure, $\mathcal{O}_{X} \otimes_{k} V$ is canonically equivariant and the Taylor map (2.1) is an equivariant homomorphism.

Corollary 5.2.3. Let $X, G, \mathcal{M}$, and $V$ be as above. The functions $n_{i n j}(x)$ and $n_{\text {surj }}(x)$ are constant along $G$-orbits. In particular, if $X=G / H$ is a homogeneous space, then $n_{\text {surj }}^{1}=n_{\text {surj }}$, and $n_{i n j}(x)=N_{\text {inj }}$, for all $x \in X$.

Proof. The diagonal map $\Delta: X \rightarrow X \times_{k} X$ is tautologically $G$-equivariant with respect to the diagonal action of $G$ on $X \times_{k} X$. Hence the canonical surjection $s: \Delta^{*}\left(\mathcal{O}_{X \times{ }_{k} X}\right) \rightarrow \mathcal{O}_{X}$, with kernel $I_{\Delta}$, is an equivariant map of $G$-equivariant sheaves. Call the isomorphism (the identity map after canonical identifications) $\alpha: d_{1}^{*} \Delta^{*}\left(\mathcal{O}_{X \times_{k} X}\right) \cong d_{0}^{*} \Delta^{*}\left(\mathcal{O}_{X \times{ }_{k} X}\right)$. Then $\alpha$ induces an equivariancy map $\alpha$ : $\operatorname{Ker} d_{1}^{*} s=d_{1}^{*}\left(I_{\Delta}\right) \rightarrow d_{0}^{*}\left(I_{\Delta}\right)=\operatorname{Ker} d_{0}^{*} s$ (recall the flatness). It clearly suffices to see that $\alpha\left(d_{1}^{*}\left(I_{\Delta}^{k}\right)\right)=d_{0}^{*}\left(I_{\Delta}^{k}\right)$ to obtain the equivariancy map $d_{1}^{*}\left(\mathcal{P}_{X / k}^{n}\right) \rightarrow d_{0}^{*}\left(\mathcal{P}_{X / k}^{n}\right)$; this, however, is clear since $\alpha$, as noted above, is an algebra homomorphism, such that $\alpha\left(d_{1}^{*}\left(I_{\Delta}\right)\right)=d_{0}^{*}\left(I_{\Delta}\right)$. To check that this induces a structure of an equivariant module is now an easy exercise, as well as proving that the Taylor map is equivariant. (This proof more generally shows that the infinitesimal thickenings of an equivariant module with respect to a $G$-subvariety are equivariant.)

The proof of the corollary is immediate from the further fact that the restriction of an equivariant module to an orbit is an equivariant module on the orbit, which is a homogeneous space $X=G / H$. For equivariant modules on homogeneous spaces there is an equivalence of categories between the category of coherent $G$ equivariant sheaves on $X$ and finite-dimensional $H$-representations ([18, 19]). In one direction this equivalence is given by taking the fibre $\mathcal{M}_{H}$, with a canonical induced $H$-action, of the module $\mathcal{M}$ in the $H$-invariant point. If $x=g^{-1} H \in X$, the stabiliser of $x$ is $H^{g}$, and the fibre $\mathcal{M}_{x}$ is an $H^{g}$-representation, and then the different representations are related by $\mathcal{M}_{x} \cong g \mathcal{M}_{H}$. This is also true for morphisms, and shows that the rank of an equivariant homomorphism is the same in each fibre over a closed point of the same orbit. This implies that the rank is the same for each point of the orbit, since $k$ is algebraically closed. In particular, an equivariant module is locally free.

In the case where $G$ is a simply-connected reductive group it is now possible to give a rough estimate of $n_{i n j}(V)=N_{i n j}$ and $n_{\text {surj }}(V)$ when $V=\Gamma(G / B, \mathcal{L}(\lambda))$, since any invertible sheaf $\mathcal{L}(\lambda)$ is $G$-equivariant on $G / B([18])$. 
Corollary 5.2.4. Let $w_{0}$ be the longest word in the Weyl group, $R$ the positive roots relative to $B$, and $\check{\alpha}$ the coroot associated to $\alpha \in R$. If $\lambda$ is a dominant weight and $\lambda-w_{0}(\lambda)=\sum_{\alpha \in R} k_{\alpha} \alpha$, denote the minimal value of $\sum_{\alpha \in R} k_{\alpha}$ by $k(\lambda)$. If $X=G / B$ and $V=\Gamma(X, \mathcal{L})$, has highest weight $\lambda$, then

$$
k(\lambda) \leq N_{i n j} \leq \sum_{\alpha \in R}\langle\lambda, \check{\alpha}\rangle .
$$

Furthermore, $\lambda-w_{0}(\lambda) \geq \sum_{\alpha \in R} k_{\alpha} \alpha$, if $\sum_{\alpha \in R} k_{\alpha} \leq n_{\text {surj }}$.

Proof. Take $x=B \in G / B$. Then

$$
H \operatorname{Hom}_{\mathcal{O}_{x}}\left(\mathcal{P}_{X / k}^{n}(\mathcal{L}(\lambda))_{x}, k\right) \cong \mathrm{U}^{n}(\mathfrak{g}) v_{\lambda} \subset \mathrm{U}(\mathfrak{g}) \otimes_{\mathrm{U}(\mathfrak{b})} k v_{\lambda},
$$

where the last module is the Verma module with highest weight vector $v_{\lambda}$, and $\mathrm{U}^{n}(\mathfrak{g})$ denotes the elements in $\mathrm{U}(\mathfrak{g})$ of order less than or equal to $n$ ([1]). Hence the dual of the fibre of the Taylor map at $x$, is the vector space homomorphism $\mathrm{U}^{n}\left(\mathfrak{u}^{-}\right) v_{\lambda} \rightarrow V_{\lambda}$, induced by the surjection of the Verma module to the irreducible module. The Taylor map is injective if and only if its dual is surjective if and only if a nonzero element of lowest weight $w_{0}(\lambda)$ is contained in the image, implying the first inequality for $N_{i n j} . F^{r}=\mathrm{U}^{r}\left(\mathfrak{u}^{-}\right) v_{\lambda}$ is a filtration of $V_{\lambda}$, and $g r V_{\lambda}$ is a $S\left(u^{-}\right)$module, and $N_{i n j}$ may be expressed as the least $n$ such that $\mathfrak{m}^{n+1} \subset \operatorname{Ann} g r V_{\lambda}$, where $\mathfrak{m}$ is the ideal generated by $\mathfrak{u}^{-}$. For the standard basis $x_{\alpha}$, the relations $x_{\alpha}^{n_{\alpha}+1} v_{\lambda}=0$, where $n_{\alpha}=\langle\lambda, \check{\alpha}\rangle$ are true ([17]), implying the second inequality. The inequality for $n_{\text {sur } j}$ is immediate.

For root systems of type $A_{1}$ and $A_{2}$ one has $k(\lambda)=N_{i n j}$ while the upper limit above for $N_{i n j}$ is strictly larger in general for $A_{2}$. It would be interesting to know the precise value in terms of the weight $\lambda$.

Hidden symmetry. Let us now consider the problem whether the above situation is in some sense the only case. We have the following result, which might be epistemologically interpreted as strengthening our general philosophy that the construction $X_{V}$ is worthwhile to pursue since it (under some conditions, of course) detects hidden geometry, in this case the underlying homogeneous space.

Proposition 5.2.5. Let $V$ be a finite-dimensional $k$-subspace in an affine ring $R=\mathcal{O}_{X_{0}}\left(X_{0}\right)$, such that $X_{0} \subset X_{V} \subset \mathbf{P}\left(V^{*}\right)$. Assume that $\mathfrak{g} \subset \mathcal{D}_{X_{0}}^{1}\left(X_{0}\right)=\mathcal{D}_{R / k}^{1}$ is a reductive Lie algebra, and that $V$ is a representation. Then the action of $\mathfrak{g}$ on $V$ may be integrated to an action of an algebraic group $G$, whose associated Lie algebra is $\mathfrak{g}$. This action may be canonically extended to $X_{V}$, in such a way that $\mathcal{L}_{V}$ is an equivariant invertible sheaf. Furthermore, $\mathfrak{g} \subset \Gamma\left(X_{V}, \mathcal{D}_{X_{V}}\left(\mathcal{L}_{V}\right)\right)$, and $V \subset \Gamma\left(X_{V}, \mathcal{L}_{V}\right)$. In the special case that $V$ is irreducible and $\mathfrak{g}$ is locally transitive on $X_{0}$ and there is a point $x_{0} \in X_{0}$, such that the kernel of $\mathfrak{g} \rightarrow \mathcal{D}_{X_{0} / k, x_{0}}$ is a parabolic subalgebra, we have $X_{V} \cong G / P$ for some parabolic subgroup $P \subset G$ and $\mathcal{L}_{V}=\mathcal{L}(\lambda)$ for some integral dominant weight $\lambda$.

Proof. The action of $\mathfrak{g}$ on $V$ extends to a semi-simple homogeneous action on $\mathrm{S}[V][t]$, such that $\gamma: \mathrm{S}[V][t] \rightarrow R[t]$ is equivariant, where $\mathfrak{g}$ acts through the inclusion $\mathfrak{g} \subset \mathcal{D}_{R / k}^{1}$ on $R[t]$ (Definition 4.1.2). Hence there is a compatible homogeneous action on $A_{V}=\operatorname{Im} \gamma(\mathrm{S}[V t])$, and this semi-simple action may be integrated to an action of a simply-connected algebraic group $G$ that has $\mathfrak{g}$ as its Lie algebra (see [16]). Hence there is a $G$-action on $X_{V}$, and it is easily checked that $\mathcal{L}_{V}$ is 
an equivariant invertible sheaf. From this follows that $\mathfrak{g} \subset \Gamma\left(X_{V}, \mathcal{D}_{X_{V}}\left(\mathcal{L}_{V}\right)\right)$, and $V \subset \Gamma\left(X_{V}, \mathcal{L}_{V}\right)$. This is the first part of the proposition. Using the additional assumptions in the second part, we get a map $g \mapsto g x_{0}, G \rightarrow X_{V}$, where the kernel has to be a parabolic subgroup $P$ of $G$. Hence we have a closed immersion $\phi: G / P \rightarrow X_{V}$. The local transitivity implies that there is an open subset $U \subset X_{0}$ that is in the image of $\phi$, and hence $\phi$ is an isomorphism. Since $V$ is a subset of the irreducible $G$-module $\Gamma\left(X_{V}, \mathcal{L}_{V}\right)$, it must equal the last module, and we are in the situation described in the beginning of the section.

\section{TORIC VARIETIES}

We continue to assume that $k$ is algebraically closed. If $V \subset k\left[x_{1}, \ldots, x_{n}\right]$ is a finite-dimensional vector space generated by monomials, $X_{V}$ will be a toric variety. Differential operators that preserve such $V$ have been considered in an affine situation in [29, 31, 30, 7], without using toric varieties. There is however much known about rings of differential operators on toric varieties - for example, systematic procedures to calculate generators - by the work of Musson [25] (see also [20, 26]), and one aim of this section is to illustrate the use of this, as well as other toric techniques. We will also calculate the injectivity order for global sections of line bundles on nonsingular complete toric varieties; we will in particular describe the locus of Weierstrass points for line bundles on Hirzebruch surfaces.

6.1. Completion of monomial vector spaces. Recall the construction of projective toric varieties; cf. 8 for details. Let $M=\mathbf{Z}^{n}$ be a lattice. For $m=$ $\left(m_{1}, \ldots, m_{n}\right) \in M$ define

$$
x^{m}=x_{1}^{m_{1}} \ldots x_{n}^{m_{n}} \in k\left[x_{1}, x_{1}^{-1}, \ldots, x_{n}, x_{1}^{-1}\right]=k[T], \quad k[x]=k\left[x_{1}, \ldots, x_{n}\right] .
$$

The torus in the nomenclature is Spec $k[T]$. If $V$ is the vector space generated by monomials $x^{m}, m \in P$, where $P \subset M$ is a convex polytope, $X_{V}$ (Section (4) is usually denoted $X(P)$ and the associated equivariant line bundle $\mathcal{L}(P)$; cf. [8, Section 1.5]. The construction of $X(P)$ is explicitly described as follows. Let $m_{i}$ be a vertex of $P$ and let $M_{i} \subset M$ be the semigroup generated by the set $\left\{p-m_{i} \mid p \in P\right\}$ (or the elements of $M$ that lie in the angle at $m_{i}$ bounded by the codimension 1 faces that meet at $m_{i}$ ). Then define $k\left[M_{i}\right] \subset k[T]$ as the algebra generated by $x^{m}$, $m \in M_{i}$, and $U_{i}=\operatorname{Spec} k\left[M_{i}\right]$. Furthermore, define $L_{i}$ as $k\left[M_{i}\right] x^{m_{i}} \subset k[T]$, with an obvious inclusion $V \rightarrow L_{i}$. These local data $U_{i}$ and $L_{i}$ glue in a way that is uniquely determined by the given inclusions into $k[T]$; this completes the construction. We also have $V=\bigoplus_{m \in P} k x^{m}=H^{0}(X(P), \mathcal{L}(P))([8,3.4])$. The line bundle $\mathcal{L}(P)$ will be very ample if and only if the following condition is satisfied:

The polyhedron $P$ is the convex hull of the points $m_{i}$, and for each $i$ the semigroup generated by the set $\left\{p-m_{i} \mid p \in P\right\}$ is saturated ([loc. cit.]).

As proven in [25] (see also [20, 26]), there are sufficiently many differential operators on an invertible very ample sheaf on a projective toric variety $X(P)$ to make $\Gamma(X, \mathcal{L}(P))$ a simple $R=\Gamma\left(X, \mathcal{D}_{\mathcal{L}}(P)\right)$-module. Hence by Theorem 3.3.6 every differential operator preserving $V$ may be decomposed as the sum of an annihilator and a global differential operator on the line bundle. 
Proposition 6.1.1. Let $X=\mathbf{A}^{n}$. Assume that $V=\bigoplus_{m \in P \cap M} k x^{m} \subset k[x]$, where $P$ is a convex polytope satisfying the very ampleness condition above. Then

(1) $V=\Gamma\left(X_{V}, \mathcal{L}_{V}\right)$, and the restriction map

$$
\Gamma\left(X_{V}, \mathcal{D}_{\mathcal{L}_{V}}\right) \rightarrow \mathcal{D}_{k[x] / k}^{V} / \text { Ann } V
$$

is surjective.

(2) The object $\left(X(P), \mathcal{O}_{X(P)}, V \cong \Gamma(X(P), \mathcal{L}(P)), \mathcal{L}(P)\right)$ is a full completion of $\left(X, \mathcal{O}_{X}, V \subset k[x], \mathcal{O}_{X}\right)$ (Definition 4.1.1).

Musson describes the ring of global differential operators explicitly as a quotient of a ring of invariants of differential operators on an open subset of the affine space (see also 20 for a procedure to calculate generators). In general this gives smooth varieties where the global ring of differential operators is not generated by first order differential operators, hence not "Lie-theoretic" in the sense of 21.

\subsection{Calculation of $n_{i n j}$ and $n_{s u r j}$ for toric varieties in the smooth case.}

Calculation of $n_{i n j}(x)$. The condition that the projective toric variety $X(P) / k$ be nonsingular is that the following basis condition holds at each vertex $m_{i}$ of the strongly convex polytope $P$ (cf. [8]): There are exactly $n$ edges $E_{i j}$ meeting at $m_{i}$, and if $e_{i j}$ is a minimal element of $M$ connecting $m_{i}$ with another point in $E_{i j}$, then the set $\left\{e_{i j}\right\}_{j=1}^{n}$ is a basis of $M$, which we call the basis at the vertex $m_{i}$. It generates $M_{i}$. Let $x$ be a point in $X(P)$ with residue field $k_{x}$. By equivariance the function $n_{i n j}(x)$ is constant along each orbit of the torus on $X(P)$. Letting $\xi_{1}, \ldots, \xi_{b}$ be the generic points of the orbits it suffices therefore to compute the numbers $n_{i n j}\left(\xi_{i}\right)$. If $\xi_{i}$ specialises to $\xi_{j}$, then $P_{j}$ is a face of $P_{i}$ and by semicontinuity, $n_{i n j}\left(\xi_{j}\right) \geq n_{i n j}\left(\xi_{i}\right)$. We put $N_{i n j}(P)=n_{i n j}(\xi)$ if $\xi$ is the generic point of $T$ (or of $X(P)$ ). These orbits are in 1-1 correspondence with the faces $P_{r}$ of the polytope. Suppose that $m_{i} \in P_{r}$, and that $P_{r}$ contains precisely the basis vectors $e_{i j}, j \in J_{r}$, in the basis at $m_{i}$. Then the orbit corresponding to $P_{r}$ is an open dense subset of the subvariety defined by the ideal $\left(x_{i}, i \notin J_{r}\right)$. It is contained in $U_{i}=\operatorname{Spec} k\left[M_{i}\right]$. Note that to the vertices of the polytope there correspond closed invariant points, and the closure of each orbit contains at least one such point. Denote $H_{r}=\left\{m_{i}+\sum_{j \in J_{r}} a_{i j} e_{i j} \mid a_{i j} \in \mathbf{N}\right\}$. Then the generic point $\xi_{r}$ of the orbit corresponding to $P_{r}$ has $k_{\xi_{r}}=k\left(x^{e_{i j}} \mid e_{i j} \in J_{r}\right)$. If $m=\sum_{j=1}^{n} m_{i j} e_{i j}$, set $d(m)=\sum_{j \notin J_{r}} m_{i j}$; note that we do not count coordinates that correspond to directions along the face $P_{r}$. In the proposition below we will consider parallel translates $H=H_{r}+m$ of $H_{r}$ at a distance $d_{H}=d(m)$ from $H_{r}$. The intersection $H \cap P \subset P$ is a strictly convex polytope, parallel to the polytope $P_{r}$, and we will relate $N_{i n j}(H \cap P)$, the generic injectivity order of the polytope $H \cap P$, to $n_{i n j}\left(\xi_{r}\right)$. We first note that the generic injectivity order $N_{i n j}(P)$ is intrinsic to $P$ and the lattice it generates.

Lemma 6.2.1. Let $P$ be a polytope in $M$ and let $N$ be the sublattice generated by $P$. The generic injectivity order of $V=\bigoplus_{m \in P} k x^{m}$ considered as a subspace of $k[M]$, denoted $N_{i n j}(P)$ above, is the same as the injectivity order of $V$ considered as a subspace of $k[N]$.

We leave out the straightforward proof. 
Proposition 6.2.2. Assume that $P$ is a strongly convex polytope satisfying the basis condition, and that $X(P) / k$ and $\mathcal{L}(P)$ are the corresponding smooth toric variety and very ample line bundle. Let $\left\{\xi_{i}\right\}$ be the generic points of the orbits of the action by the torus.

(1)

$$
\begin{aligned}
& n_{i n j}\left(\xi_{r}\right)=\max \{ N_{i n j}(H \cap P)+d_{H} \\
&\left.\mid H=m+H_{r} \text { is a parallel translate of } H_{r}, H \cap P \neq \emptyset\right\} .
\end{aligned}
$$

(2) Let $P^{v}$ be the set of vertices in $P$ and $\xi_{i}$ be a closed orbit, i.e., a closed point, with corresponding vertex $m_{i}$ in $P^{v}$, then

$$
n_{i n j}\left(\xi_{i}\right)=\max \left\{\sum_{j=1}^{n} m_{i, j} \mid m \in P^{v}\right\}
$$

(the maximal distance from $m_{i}$ to any other vertex).

$$
n_{i n j}(V)=\max \left\{\max \left\{\sum_{j=1}^{n} m_{i, j} \mid m \in P^{v}\right\} \mid m_{i} \in P^{v}\right\}
$$

(the maximal distance between pairs of vertices of $P$ ).

Proof. (1) Identifying $k_{\xi_{r}} \otimes_{\mathcal{O}_{\xi_{r}}} \mathcal{L}_{\xi_{r}}=k_{\xi_{r}}$ and $\mathcal{D}_{X(P) / k, \xi_{r}}(\mathcal{L})=\mathcal{D}_{X / k, \xi_{r}}$ we want to determine the smallest integer $n_{i n j}\left(\xi_{r}\right)$ such that $l \geq n_{i n j}\left(\xi_{r}\right)$ implies that the evaluation map

$$
W_{x}^{l}: k_{\xi_{r}} \otimes_{\mathcal{O}_{x}} \mathcal{D}_{X / k, \xi_{r}}^{l} \rightarrow \operatorname{Hom}_{k}\left(\bigoplus_{m \in P} k x^{m}, k_{\xi_{r}}\right)
$$

is surjective (Theorem 3.2.1). Let $\phi_{m} \in \bigoplus_{m \in P} \operatorname{Hom}_{k}\left(k x^{m}, k_{\xi_{r}}\right)$ be defined by $\phi_{m}\left(x^{m^{\prime}}\right)=\delta_{m, m^{\prime}} \in k_{\xi_{r}}$. That $n \geq n_{i n j}\left(\xi_{r}\right)$ means that $\phi_{m} \in \operatorname{Im} W_{\xi_{r}}^{n}$ for each $m \in P$. Let $P_{r}$ be the face of $P$ corresponding to $\xi_{r}$, assume that the vertex $m_{i} \in P_{r}$ and set $\left\{x_{1}, \ldots, x_{d}\right\}=\left\{x^{e_{i j}} \mid e_{i j} \in J_{r}\right\}$, while $\left\{x_{d+1}, \ldots, x_{n}\right\}$ correspond to basis vectors that are not parallel to $P_{r}$. Let $\partial_{i} \in \mathcal{D}_{k[T] / k}$ be defined by $\partial_{i}\left(x_{i}\right)=\delta_{i j}$. Put furthermore, $H_{r}=m_{i}+\sum_{j \in J_{r}} \mathbf{N} e_{i j}$, so $P_{r} \subset H_{r}$. The residue field of the orbit is $k_{F}:=k\left(x_{1}, \ldots, x_{d}\right)$. Let $k\left[\partial^{(1)}\right]=k\left[\partial_{1}, \ldots, \partial_{d}\right]$ and $k\left[\partial^{(2)}\right]=k\left[\partial_{d+1}, \ldots, \partial_{n}\right]$. For $s \in P$ there is a unique decomposition $x^{s}=x^{m(s)} x^{f(s)}$, where $f(s) \in H_{r}$, $m(s) \in \sum_{j \notin J_{r}} m_{i j}(s) e_{i j}$, and the differential operator $\partial^{m(s)}:=\prod_{j \notin J_{r}}\left(\partial_{j}\right)^{m_{i, j}(s)} \in$ $k\left[\partial^{(2)}\right]$ satisfies $\partial^{m(s)}\left(x^{s}\right)=C x^{f} \in k_{F}$ for some $0 \neq C \in k$. Note also that $\operatorname{Im} W\left(k_{F} \otimes_{k} k\left[\partial^{(1)}\right] \partial^{m}\right)\left(x^{s}\right)=0$ if $m \neq m(s)$, so that $\operatorname{Im} W\left(k_{F} \otimes_{k} k[\partial]\right)\left(x^{s}\right)=$ $k_{F} \otimes_{k} k\left[\partial^{(1)}\right] \partial^{m(s)}\left(x^{s}\right)$. Hence for any operator $Q_{s}$ such that $W\left(Q_{s}\right) x^{t}=\delta_{t, s}$, there exists $Q_{s}^{1} \subset k_{F} \otimes_{k} k\left[\partial^{(1)}\right]$ such that $W\left(Q_{s}\right)=W\left(Q_{s}^{1} \partial^{m(s)}\right)$ and $W\left(Q_{s}^{1}\right) x^{f}=\delta_{f, f(s)}$; conversely, assuming the last relation for some $Q_{s}^{1}$ we get that $W\left(Q_{s}^{1} \partial^{m(s)}\right) x^{t}=$ $\delta_{t, s}$. If $o(Q)$ denotes the order of a differential operator, and we assume $Q_{s}$ has minimal order such that $W\left(Q_{s}\right)=\phi_{s}$, we have $o\left(Q_{s}\right)=o\left(Q_{s}^{1}\right)+m(s)$. If $H=$ $m+H_{r}$ is a parallel translate of $H_{r}$, then $s \in H \cap P$ iff $f(s) \in H \cap P-m$ and hence $\left\{W\left(Q_{s}^{1}\right) \mid s \in H \cap P\right\}$ clearly is a $k_{F}$-basis of $\operatorname{Hom}\left(\sum_{p \in H \cap P-m} k x^{p}, k_{F}\right)$. Hence $\max \left\{l \mid s \in H \cap P\right.$ and $\left.\phi_{s} \in \operatorname{Im} W_{\xi_{r}}^{l}\right\}=N_{i n j}(H \cap P-m)+d_{H}$, and since $N_{i n j}(Q-m)=N_{i n j}(Q)$ for any polytope $Q$ in $M$, this completes the proof of (1). 
(2) This follows immediately from (1) since $H_{i}$ is a point.

(3) This follows from (2), since $n_{i n j}(V)=\max \left\{n_{i n j}\left(\xi_{i}\right) \mid m_{i} \in P^{v}\right\}$.

Proposition 6.2.2 reduces the problem to the computation of $N_{i n j}=N_{i n j}(P)$ $\left(=N_{i n j}(V)\right)$ when $P$ is a strongly convex polytope satisfying the basis condition. Letting $K=k\left(x_{1}, \ldots, x_{n}\right)$ be the function field of $X(P)$ we can regard $V$ as a subset of $K$. We have $\mathcal{D}_{X / k, \xi}=\mathcal{D}_{K / k}=K\left[\nabla_{1}, \ldots, \nabla_{n}\right]$, where $\nabla_{i}$ are derivations defined by $\nabla_{i}\left(x_{j}\right)=\delta_{i j} x_{i}$, and we put $A=k\left[\nabla_{1}, \ldots, \nabla_{n}\right] \subset K\left[\nabla_{1} \ldots, \nabla_{n}\right]$; put also $A_{l}=\{P \in A \mid \operatorname{deg} P \leq l\}$, where deg is the ordinary degree of a polynomial. Clearly, $A$ preserves each subspace $k x^{m} \subset V$, so $W^{l}$ (defined in 2.1) restrict to diagonal maps

$$
\bar{W}^{l}: A_{l} \rightarrow \operatorname{End}_{k}^{d}(V)=\bigoplus_{m \in P} \operatorname{Hom}_{k}\left(k x^{m}, k x^{m}\right) \cong k^{|P|} .
$$

They are compatible and define a map $W: A \rightarrow \operatorname{End}_{k}^{d}(V)$ (loc. cit). If $I_{l}$ is the image of $\bar{W}^{l}$, it follows easily that $K I_{l}$ is the image of $W^{l}$; hence $\operatorname{rk} \bar{W}^{l}=\operatorname{rk} W^{l}$. Putting $J_{P}=\operatorname{Ker} \bar{W}=\operatorname{Ann} V \cap A$ we get $\operatorname{rk} W^{l}=\operatorname{rk} \bar{W}^{l}=\operatorname{dim} A /\left(J_{P}+\mathfrak{m}^{l+1}\right)$ where $\mathfrak{m}=\left(\nabla_{1}, \ldots, \nabla_{n}\right)$. In particular,

$$
N_{i n j}(P)=\min \left\{l \mid \frac{A}{J_{P}+\mathfrak{m}^{l+1}}=\frac{k[\nabla]}{J_{P}+\mathfrak{m}^{l+2}}\right\} .
$$

(Recall that $\operatorname{rk} W^{0}<\operatorname{rk} W^{1}<\cdots<\operatorname{rk} W^{l} \cdots<\operatorname{rk}^{N_{i n j}(P)}=|P|$; see the proof of Proposition 3.1.1) The above expression for $\mathrm{rk} W^{l}$ was first given in 28, using the explicit form of the matrix of the Taylor map $d_{V}^{l}$ in the natural bases.

If $Z$ is a line in $M$, we let $|P \cap Z|$ be the number of points in $P \cap Z$. Put $d^{g}(P)=\max \{|P \cap Z| \mid \mathrm{Z}$ a line in $M\}$. By Proposition 3.1.1 $N_{i n j}(P) \leq|P|$, and Proposition 6.2.2 implies that $N_{i n j}(P)$ is actually bounded above by the maximal distance between the vertices of $P$ (computed in bases at the different vertices).

Proposition 6.2.3. Let $P$ be a subset of $M$. Then $d^{g}(P)-1 \leq N_{i n j}(P)$.

Proof. Let $Z$ be a line in $M$ such that $|Z \cap P|=d^{g}(P)$. Choose a point $m \in Z \cap P$ and let $P_{m} \in k[\nabla]$ satisfy $P_{m} x^{m^{\prime}}=\delta_{m m^{\prime}}$. Then $P_{m}$ has a nonzero reduction $P_{m}^{A}$ in $A=k[\nabla] / I_{Z}$, where $I_{Z}$ is the ideal of $Z$, and $P_{m}^{A}$ vanishes at $d^{g}(P)-1$ points along $Z$; hence $\operatorname{deg} P_{m} \geq \operatorname{deg} P_{m}^{A} \geq d^{g}(P)-1$. This implies $N_{i n j} \geq d^{g}(P)-1$.

Calculation of $n_{\text {surj }}$. Let $P$ be a strongly convex polytope. The length $l_{i j}$ of an edge $E_{i j}$, connecting the vertices $m_{i}$ and $m_{j}$ is the integer such that $m_{j}-m_{i}=l_{i j} e_{i j}$ (see above for the definition of $e_{i j}$ ). Denote by $s(P)$ the minimal length of an edge ( $P$ is $s(P)$-convex).

For a smooth and proper toric variety, Di Rocco [5] has proved that $\mathcal{L}(P)$ is $s$-jet ample if and only if $s(P) \geq s$, for $X(P)$ smooth and proper. This implies immediately that $s(P) \geq n_{\text {surj }}$. Her method is to use Cox's homogeneous coordinate ring to describe the toric variety; this is not necessary in our simpler case. We do not give a complete computation of the function $x \mapsto n_{\text {surj }}(x)$, the surjectivity order at $x$, since we do not need it, but it should be clear that it is locally constant on each orbit of the torus, and may be estimated accordingly.

Proposition 6.2.4. (1) Suppose that $P \subset M$ is a strongly convex polytope satisfying the basis condition. Then $n_{\text {surj }}=s(P)$ and hence equals the degree of jet-ampleness. 
(2) Let $P$ be a strictly convex polytope. Then $n_{\text {surj }}^{1}=\min \left\{n^{1}(F) \mid F\right.$ a codimension 1 face of $P$ \}.

Proof. (1) $X(P)$ is smooth if and only if any semigroup $M_{i}$ is generated by a basis of $M$. Fix $i$ and let $k\left[M_{i}\right] \cong k\left[x_{1}, \ldots, x_{n}\right]=: k[x]$. The principal parts for $k[x]$ may be described as $d: k[x] \otimes_{k} V_{l} \stackrel{\sim}{\longrightarrow} \mathcal{P}_{\mathbf{A}^{n} / k}^{l}$, where $V_{l}$ is the vector space generated by all monomials in $x$ with total degree at most $l$, and the map is the Taylor map. Recalling that $L(P) x^{-m_{i}}=k\left[M_{i}\right]$, the $l$ th Taylor map restricted to $U_{i}$ may be described in $U_{i}$, as

$$
k\left[M_{i}\right] \otimes V x^{-m_{i}} \rightarrow \mathcal{P}_{U_{i} / k}^{l} .
$$

By convexity, $P$ will contain all $\sum_{m=1}^{n} a_{i j_{m}} e_{i j_{m}}+m_{i}$ where $\sum_{j} a_{i j_{m}} \leq s=s(P)$. In particular, $V_{s} \subset V x^{-m_{i}}$, and by the description of $\mathcal{P}_{U_{i} / k}^{s}$ this implies that the $s$ th Taylor map is surjective. Conversely, assume that the $s$ th Taylor map is surjective. Tensoring with $k[x] /(x)$, we obtain that $V x^{-m_{i}} \rightarrow k[x] /(x)^{s+1} \rightarrow k\left[x_{m}\right] /\left(x_{m}\right)^{s}$ is surjective. This gives that $\left\{1, x_{m}, \ldots, x_{m}^{s}\right\} \subset V x^{-m_{i}}$, which is, interpreted in terms of $P$, precisely the condition that $s(P)=s$.

(2) Again the Taylor map is an equivariant homomorphism between $T$-homogeneous sheaves and so the support of the cokernel and kernel will be unions of closures of orbits under the torus $T$. Hence to check the surjectivity in codimension 2 , it suffices to check surjectivity at the orbits of codimension 0 or 1 . In fact, it suffices to prove surjectivity for all orbits of codimension precisely 1 , since the support is closed and the only open orbit contains all codimension 1 orbits in its closure.

Corollary 6.2.5. Assume the conditions in Proposition 6.1.1. If $m \leq s(P)$, then the restriction map gives an isomorphism

$$
\Gamma\left(X_{V}, \mathcal{D}^{m}\left(\mathcal{L}_{V}\right)\right) \rightarrow \mathcal{D}_{k[x] / k}^{m, V} .
$$

Proof. This is immediate from Corollary 3.3.5.

Hirzebruch surfaces. We exemplify with line bundles on Hirzebruch surfaces. The polytope is in this case determined by the finite-dimensional vector space of polynomials

$$
V_{k l}^{r}:=\left\langle x^{i} y^{j} \mid \quad 0 \leq i+r j \leq k, 0 \leq j \leq l\right\rangle,
$$

where $r, k, l$ are nonzero integers and $r \geq 1$. We will restrict ourselves to the truncated case $k-l r \geq 0$. As noted already in [10], this vector space is the restriction of the global sections of the equivariant line-bundle $\mathcal{O}_{\Sigma_{r}}(k, l)$ on the Hirzebruch surface $\Sigma_{r}$ to the affine space $\mathbf{A}^{2} \subset \Sigma_{r}$. The differential operators of order 1 that preserve the vector space are described in [loc. cit], and in [7] a graphic method is given to calculate the higher order differential operators that preserve $V$. This graphic method is just a use of the obvious bigrading, and as such a special case of the much more powerful methods of Jones/Musson. Even in the special case of Hirzebruch surfaces, the methods of the latter authors give fuller information on the whole ring of differential operators.

In particular, it is known that $\mathcal{D}^{V_{k l}^{r}}$ is not generated by differential operators of order less than 1 . Let us see what the preceding theory and the literature on toric varieties tells us. The vertices of the polytope are $V_{1}=(k, 0), V_{2}=(0,0), V_{3}=$ $(0, l), V_{4}=(k-l r, l)$; denote the edge between the first two vertices by $E_{1}$, between the second and third by $E_{2}$, and so on. 
Proposition 6.2.6. Let $k-l r \geq 0$. Then

(1)

$$
X=X_{V_{k l}^{r}}=\Sigma_{r}=\mathbf{P}\left(\mathcal{O}_{\mathbf{P}^{1}} \oplus \mathcal{O}_{\mathbf{P}^{1}}(r)\right)
$$

and $\mathcal{L}_{V_{k l}^{r}}=\mathcal{O}_{\Sigma_{r}}(l, k)$.

(2) $n_{\text {surj }}(X)=n_{\text {surj }}^{1}(X)=\operatorname{Min}\{l, k-l r\}$.

(3) $N_{i n j}=k, n_{i n j}\left(V_{1}\right)=k+l, n_{i n j}\left(V_{2}\right)=k, n_{i n j}\left(V_{3}\right)=k+l, n_{i n j}\left(V_{4}\right)=k+l$, $n_{i n j}\left(E_{1}\right)=k, n_{i n j}\left(E_{2}\right)=k, n_{i n j}\left(E_{3}\right)=k+l, n_{i n j}\left(E_{4}\right)=k$. In particular, $n_{\text {inj }}=k+l$.

(4) The Weierstrass subsets (see (3.2)) are $W\left(V_{k l}^{r}\right)=W_{l}\left(V_{k l}^{r}\right)=\mathbf{P}^{1}$ where $\mathbf{P}^{1}$ is the closure of the orbit of the edge $E_{3}$.

(5) The ring $\mathcal{D}_{k l}^{V_{k l}^{r}}$ is given up to Ann $V_{k l}^{r}$ by the differential operators

$$
\begin{aligned}
& R=\Gamma\left(\Sigma_{r}, \mathcal{D}(\mathcal{L})\right)=k\left[\partial_{x}, x^{j} \partial_{y}, x \pi, \partial_{x}^{j} y\left(\nabla_{y}\right) \pi(\pi+1)\right. \\
& \left.\ldots(\pi+r-j-1), x \partial_{x}, y \partial_{y} \mid j=0,1 \ldots, r\right] . \\
& \text { Here } \pi:=x \partial_{x}+r y \partial_{y}-k \text { and } \nabla_{y}:=y \partial_{y}-l .
\end{aligned}
$$

Proof. (1) See [5, 8 .

(2) The length of the edges are $k, l, k-l r, l$, and hence by Proposition 6.2.4. $n_{\text {surj }}\left(V_{k l}^{r}\right)=\operatorname{Min}\{l, k-l r\}$. Next consider $n_{\text {surj }}^{1}\left(V_{k l}^{r}\right)$. At the edge $E_{1}$, local coordinates are $x=x^{(1,0)}$ and $y=x^{(0,1)}$, and $p\left(F_{1}\right)$ is defined by $x=1, y=0$. It is easy to see that $V_{k l}^{r} \rightarrow k[x, y] /(x-1, y)^{s+1}$ is surjective if and only if $s \leq l$, since in this case $y^{s}$ must be in the image. In the same way $n^{1}\left(E_{2}\right)=l, n^{1}\left(E_{3}\right)=l$ and $n^{1}\left(E_{4}\right)=k-l r$. Hence $n_{1}=n_{\text {surj }}\left(V_{k l}^{r}\right)=\operatorname{Min}\{l, k-l r\}$.

(3) Apply Proposition 6.2.2 to get the values along orbits of codimension $\geq 1$ (edges and points of $P$ ), noting that $N_{i n j}(Q)=|Q|-1$ if $Q \subset M$ belongs to a line in $M$. Proposition 6.2.3 gives $N_{i n j} \geq d^{g}(P)-1=k$, and since $n_{i n j}\left(V_{1}\right)=k$, by semi-continuity, we have $N_{i n j}=k$.

(4) This follows from (3).

(5) Since this is a toric situation, we know by Musson that $V:=V_{k l}^{r}$ is an irreducible module over the finitely generated and Noetherian algebra of differential operators $\Gamma\left(X_{V}, \mathcal{D}_{L}\right)$. Hence Theorem 3.3.6 applies. Finally, an explicit description of a set of generators of global differential operators on $X$ is given in 20. The restriction to $U_{1}=\operatorname{Spec} k[x, y]$ of the global differential operators on the structure sheaf $\mathcal{L}=\mathcal{O}_{\Sigma_{r}}$ are calculated to be

$$
\begin{aligned}
R=\Gamma\left(\Sigma_{r}, \mathcal{D}(\mathcal{L})\right)=k\left[\partial_{x}, x^{j} \partial_{y}, x \pi, \partial_{x}^{j} y\left(\nabla_{y}\right) \pi(\pi+1)\right. \\
\left.\ldots(\pi+r-j-1), x \partial_{x}, y \partial_{y} \mid j=0,1 \ldots, r\right] .
\end{aligned}
$$

Here $\pi:=x \partial_{x}+r y \partial_{y}$ and $\nabla_{y}=y \partial_{y}$. One may either repeat these calculations for an arbitrary line-bundle - in Jones and Musson's framework this is an easy, if laborious exercise - or one may use [20. Theorem 4.9], to see that redefining $\pi:=x \partial_{x}+r y \partial_{y}-k$, and also $\nabla_{y}:=y \partial_{y}-l$ in the expression

$$
P(j)=\partial_{x}^{j} y\left(\nabla_{y}\right) \pi(\pi+1) \ldots(\pi+r-j-1),
$$

will give that the above expression for the ring of differential operators is valid for the line-bundle $\mathcal{L}=\mathcal{L}_{V_{k l}^{r}}$. This follows since after redefinition the differential operators on the right-hand side are easily seen to act on $\mathcal{L}$, and the associated graded rings to the filtration by differential operator order are equal. 


\section{REFERENCES}

[1] A. Beilinson and J. Bernstein, Localisation de g-modules, C. R. Acad. Sci. Paris Sér. I Math., 292 (1981), pp. 15-18. MR0610137 (82k:14015)

[2] J. Bochnak, M. Coste, And M.-F. Roy, Géométrie algébrique réelle, vol. 12 of Ergebnisse der Mathematik und ihrer Grenzgebiete (3) [Results in Mathematics and Related Areas (3)], Springer-Verlag, Berlin, 1987. MR0949442 (90b:14030)

[3] A. Borel, P.-P. Grivel, B. Kaup, A. Haefliger, B. Malgrange, and F. Ehlers, Algebraic D-modules, vol. 2 of Perspectives in Mathematics, Academic Press Inc., Boston, MA, 1987. MR0882000 (89g:32014)

[4] N. BourBAKI, Commutative algebra, Hermann, 1972. MR 0360549 (50:12997)

[5] S. Di Rocco, Generation of k-jets on toric varieties, Math. Z., 231 (1999), pp. 169-188. MR:1696762 (2000m:14007)

[6] J. Dixmier, Algèbres enveloppantes, Gauthiers-Villars, 1974. MR0498737 (58:16803a)

[7] F. Finkel and N. Kamran, The Lie algebraic structure of differential operators admitting invariant spaces of polynomials, Adv. in Appl. Math., 20 (1998), pp. 300-322. MR1618427 (2000g:17027)

[8] W. Fulton, Introduction to toric varieties, vol. 131 of Annals of Mathematics Studies, Princeton University Press, Princeton, NJ, 1993. The William H. Roever Lectures in Geometry. MR:1234037 (94g:14028)

[9] W. Fulton, S. Kleiman, R. Piene, and H. Tai, Some intrinsic and extrinsic characterizations of the projective space, Bull. Soc. Math. France, 113 (1985), pp. 205-210. MR0820319 (87a:14012)

[10] A. González-López, J. C. Hurtubise, N. Kamran, and P. J. Olver, Quantification de la cohomologie des algèbres de Lie de champs de vecteurs et fibrés en droites sur des surfaces complexes compactes, C. R. Acad. Sci. Paris Sér. I Math., 316 (1993), pp. 1307-1312. MR.1226121 (94h:32047)

[11] A. GonzÁlez-López, N. Kamran, and P. J. Olver, Quasi-exact solvability, in Lie algebras, cohomology, and new applications to quantum mechanics (Springfield, MO, 1992), vol. 160 of Contemp. Math., Amer. Math. Soc., Providence, RI, 1994, pp. 113-140. MR1277379 (95g:81023)

[12] A. González-López, N. Kamran, and P. J. Olver, Quasi-exact solvability in the real domain, in Field theory, integrable systems and symmetries (Quebec, PQ, 1995), Univ. Montréal, Montreal, QC, 1997, pp. 58-70. MR1482533 (99b:17029)

[13] A. Grothendieck, Éléments de géométrie algébrique. II. Étude globale élémentaire de quelques classes de morphismes, Inst. Hautes Études Sci. Publ. Math., (1961), p. 222. MRMR0217084 (36:177b)

[14] A. Grothendieck, Éléments de géométrie algébrique. IV. Étude locale des schémas et des morphismes de schémas IV, Inst. Hautes Études Sci. Publ. Math. No., 32 (1967), p. 361. MR0238860 (39:220)

[15] R. Hartshorne, Algebraic Geometry, Springer, 1885. MR0463157 (57:3116)

[16] J. E. Humphreys, Linear algebraic groups, Springer-Verlag, New York, 1975. Graduate Texts in Mathematics, No. 21. MR0396773 (53:633)

[17] _ Introduction to Lie algebras and representation theory, vol. 9 of Graduate Texts in Mathematics, Springer-Verlag, New York, 1978. Second printing, revised. MR0499562 (81b:17007)

[18] B. IvERSEN, The geometry of algebraic groups, Advances in Math., 20 (1976), pp. 57-85. MR0399114 (53:2965)

[19] J. C. Jantzen, Representations of algebraic groups, vol. 131 of Pure and Applied Mathematics, Academic Press Inc., Boston, MA, 1987. MR0899071 (89c:20001)

[20] A. G. Jones, Rings of differential operators on toric varieties, Proc. Edinburgh Math. Soc. (2), 37 (1994), pp. 143-160. MR.1258039 (95d:16030)

[21] N. Kamran, R. Milson, and P. J. Olver, Invariant modules and the reduction of nonlinear partial differential equations to dynamical systems, Adv. Math., 156 (2000), pp. 286-319. MR:1808246 (2001m:58078)

[22] M. KASHIWARA, Representation theory and D-modules on flag varieties, Société Math. de France, Astérisque, (1989), pp. 55-190. MR1021510(90k:17029) 
[23] D. Laksov and A. Thorup, Weierstrass points on schemes, J. Reine Angew. Math., 460 (1995), pp. 127-164. MR1316575 (96b:14016)

[24] D. Mumford, J. Fogarty, And F. KirWan, Geometric invariant theory, vol. 34 of Ergebnisse der Mathematik und ihrer Grenzgebiete (2) [Results in Mathematics and Related Areas (2)], Springer-Verlag, Berlin, third ed., 1994. MR:1304906 (95m:14012)

[25] I. M. Musson, Differential operators on toric varieties, J. Pure Appl. Algebra, 95 (1994), pp. 303-315. MR.1295963 (95i:16026)

[26] I. M. MusSON AND M. VAN DEN BERGH, Invariants under tori of rings of differential operators and related topics, Mem. Amer. Math. Soc., 136 (1998), pp. viii+85. MR1446212 (99i:16051)

[27] R. H. OGawa, On the points of Weierstrass in dimensions greater than one, Trans. Amer. Math. Soc., 184 (1973), pp. 401-417. MR0325997 (48:4343)

[28] D. Perkinson, Inflections of toric varieties, Michigan Math. J., 48 (2000), pp. 483-515. Dedicated to William Fulton on the occasion of his 60th birthday. MR1786502 (2001h:14066)

[29] G. Post And A. Turbiner, Classification of linear differential operators with an invariant subspace of monomials, Russian J. Math. Phys., 3 (1995), pp. 113-122. MR1337491 (96f:34014)

[30] A. TURBINER, Lie-algebras and linear operators with invariant subspaces, in Lie algebras, cohomology, and new applications to quantum mechanics (Springfield, MO, 1992), vol. 160 of Contemp. Math., Amer. Math. Soc., Providence, RI, 1994, pp. 263-310. MR1277386 $(95 \mathrm{~m}: 81070)$

[31] A. V. Turbiner, Quasi-exactly-solvable problems and sl(2) algebra, Comm. Math. Phys., 118 (1988), pp. 467-474. MR0958807 (89j:58051)

[32] M. VAn den Bergh, Some generalities on G-equivariant quasi-coherent $\mathcal{O}_{X}$ - and $D_{X}$ modules. Available at http://alpha.luc.ac.be/Research/Algebra/Publications/Geq.ps.

Department of Mathematics, Stockholm University, S-106 91 Stockholm, Sweden

E-mail address: rikard@matematik.su.se

Department of Mathematics, University of Gävle, S-801 76 Gävle, Sweden

E-mail address: rkm@hig.se 